\title{
Loading history changes the morphology and compressive force-induced expression of receptor activator of nuclear factor kappa B ligand/osteoprotegerin in MLO-Y4 osteocytes
}

\author{
Ziyi Wang ${ }^{1,2}{ }$, Yao Weng ${ }^{3}$, Yoshihito Ishihara ${ }^{1}$, Naoya Odagaki ${ }^{1}$, Ei Ei Hsu Hlaing ${ }^{1}$, Takashi Izawa ${ }^{1}$, Hirohiko \\ Okamura $^{3}$, Hiroshi Kamioka ${ }^{\text {Corresp. } 1}$ \\ ${ }^{1}$ Department of Orthodontics, Okayama University Graduate School of Medicine, Dentistry, and Pharmaceutical Sciences, Okayama University, Okayama, \\ Japan \\ 2 Research Fellow of Japan Society for the Promotion of Science, Tokyo, Japan \\ 3 Department of Oral Morphology, Okayama University Graduate School of Medicine, Dentistry, and Pharmaceutical Sciences, Okayama University, \\ Okayama, Japan \\ Corresponding Author: Hiroshi Kamioka \\ Email address: kamioka@md.okayama-u.ac.jp
}

Background: In this study, we investigated the effect of the mechanical loading history on the expression of receptor activator of nuclear factor kappa B ligand (RANKL) and osteoprotegerin (OPG) in MLO-Y4 osteocyte-like cells.

Methods: Three hours after MLO-Y4 osteocytes were seeded, a continuous compressive force (CCF) of 31 dynes $/ \mathrm{cm}^{2}$ with or without additional CCF (32 dynes $/ \mathrm{cm}^{2}$ ) was loaded onto the osteocytes. After $36 \mathrm{~h}$, the additional CCF (loading history) was removed for a recovery period of $10 \mathrm{~h}$. The expression of RANKL, OPG, RANKL/OPG ratio, cell numbers, viability, and morphology were time-dependently examined at 0,3 , 6 , and $10 \mathrm{~h}$. Then, the same additional CCF was applied again for $1 \mathrm{~h}$ to all osteocytes with or without the gap junction inhibitor to examine the expression of RANKL, OPG, the RANKL/OPG ratio, and other genes that essential to characterize the phenotype of MLO-Y4 cells. Fluorescence recovery after photobleaching (FRAP) technique was also applied to test the differences of gap-junctional intercellular communications (GIIC) among MLO-Y4 cells.

Results: The expression of RANKL and OPG by MLO-Y4 osteocytes without a loading history was dramatically decreased and increased, respectively, in response to the 1-h loading of additional weight. However, the expression of RANKL, OPG, and the RANKL/OPG ratio were maintained at the same level as in the control group in the MLO-Y4 osteocytes with a loading history but without gap junction inhibitor treatment. Treatment of loading history significantly changed the capacity of GJIC and protein expression of connexin 43 ( $\mathrm{C} \times 43$ ) but not the mRNA expression of $\mathrm{Cx} 43$. No significant difference was observed in the cell number or viability between the MLO-Y4 osteocyte-like cells with and without a loading history or among different time checkpoints during the recovery period. The cell morphology showed significant changes and was correlated with the expression of OPG, Gja1, and Dmp1 during the recovery period.

Conclusion: Our findings indicated that the compressive force-induced changes in the RANKL/OPG expression could be habituated within at least $11 \mathrm{~h}$ by 36-h CCF exposure. GJIC and cell morphology may play roles in response to loading history in MLO-Y4 osteocyte-like cells. 
1 Title: Loading history changes the morphology and compressive force-induced expression of

2 receptor activator of nuclear factor kappa B ligand/osteoprotegerin in MLO-Y4 osteocytes

\section{Running Title: Habituation of osteocytes in vitro}

4 Authors: Ziyi Wang ${ }^{1,2}$, Yao Weng ${ }^{3}$, Yoshihito Ishihara ${ }^{1}$, Naoya Odagaki ${ }^{1}$, Ei Ei Hsu Hlaing ${ }^{1}$,

5 Takashi Izawa ${ }^{1}$, Hirohiko Okamura ${ }^{3}$, Hiroshi Kamioka ${ }^{1 *}$

\section{Affiliation:}

7 1. Department of Orthodontics, Okayama University Graduate School of Medicine, Dentistry

8 and Pharmaceutical Sciences, Okayama, Japan.

9 2. Research Fellow of Japan Society for the Promotion of Science.

10 3. Department of Oral Morphology, Okayama University Graduate School of Medicine, 11 Dentistry and Pharmaceutical Sciences, Okayama, Japan.

\section{2 *Corresponding author}

13 Hiroshi Kamioka

14 Department of Orthodontics,

15 Okayama University Graduate School of Medicine, Dentistry, and Pharmaceutical Sciences

16 2-5-1 Shikata-cho, Okayama City, Okayama, 700-8558, JAPAN

17 Tel: +81862356690 / Fax: +81862356694

18 E-mail: kamioka@md.okayama-u.ac.jp 
19 Abstract

20 Background: In this study, we investigated the effect of the mechanical loading history on the 21 expression of receptor activator of nuclear factor kappa B ligand (RANKL) and osteoprotegerin 22 (OPG) in MLO-Y4 osteocyte-like cells.

23 Methods: Three hours after MLO-Y4 osteocytes were seeded, a continuous compressive force 24 (CCF) of 31 dynes $/ \mathrm{cm}^{2}$ with or without additional CCF (32 dynes $/ \mathrm{cm}^{2}$ ) was loaded onto the osteocytes. After $36 \mathrm{~h}$, the additional CCF (loading history) was removed for a recovery period of 10 h. The expression of RANKL, OPG, RANKL/OPG ratio, cell numbers, viability, and morphology were time-dependently examined at $0,3,6$, and $10 \mathrm{~h}$. Then, the same additional CCF was applied again for $1 \mathrm{~h}$ to all osteocytes with or without the gap junction inhibitor to examine the expression of RANKL, OPG, the RANKL/OPG ratio, and other genes that essential to characterize the phenotype of MLO-Y4 cells. Fluorescence recovery after photobleaching (FRAP) technique was also applied to test the differences of gap-junctional intercellular communications (GJIC) among MLO-Y4 cells.

Results: The expression of RANKL and OPG by MLO-Y4 osteocytes without a loading history was dramatically decreased and increased, respectively, in response to the 1-h loading of additional weight. However, the expression of RANKL, OPG, and the RANKL/OPG ratio were maintained at the same level as in the control group in the MLO-Y4 osteocytes with a loading history but without gap junction inhibitor treatment. Treatment of loading history significantly changed the capacity of GJIC and protein expression of connexin 43 (Cx43) but not the mRNA expression of Cx43. No significant difference was observed in the cell number or viability between the MLOY4 osteocyte-like cells with and without a loading history or among different time checkpoints during the recovery period. The cell morphology showed significant changes and was correlated with the expression of OPG, Gja1, and Dmp1 during the recovery period. 
43 Conclusion: Our findings indicated that the compressive force-induced changes in the

44 RANKL/OPG expression could be habituated within at least $11 \mathrm{~h}$ by $36-\mathrm{h}$ CCF exposure. GJIC

45 and cell morphology may play roles in response to loading history in MLO-Y4 osteocyte-like 46 cells. 


\section{Introduction}

48 Osteocytes are the most abundant (90\%-95\% of total bone cells in the adult skeleton) and long-

49 lived cell type in bone, which are major regulators of bone mechanosensation and

50 mechanotransduction (Wang et al., 2019; Qin et al., 2020). It has been proposed that the bone cell

51 network stores its mechanical loading history, allowing it to adjust its sensitivity to additional

52 mechanical loading or strain (Turner et al., 2002).

The loading history reshapes the morphology of trabecular bone and the extracellular

matrix surrounding osteocytes, as is described in the well-known Wolff's law (Kerschnitzki et al., 2013), which is also known as bone adaptation. The morphology of the extracellular matrix surrounding osteocytes can influence the extracellular fluid-flow shear stress, thereby enhancing the influence of the loading history on the bone (Kamioka et al., 2012). Consequently, the osteocyte networks are acclimated to daily mechanical stimuli in which high-magnitude strain occurs rarely, and low-magnitude signals occur much more often to accommodate a daily mechanical loading environment (Fritton, J. McLeod \& Rubin, 2000). Previous observations showed that extending the loading duration has a diminishing effect on further bone adaptation, and accommodating to a mechanical loading environment in bone cells makes them less responsive to routine or customary loading signals (Turner \& Pavalko, 1998; Robling, 2012).

Xenopus cardiomyocytes and epidermal cell progenitors can be designed by an evolutionary algorithm in silico and engineered to move in desired patterns, such as drawing a circle or writing English letters (Kriegman et al., 2020). However, the lacuna-canalicular system and bone cell network are more complicated than these artificial organisms. Therefore, the bone cell network is considered to be encoded with a unique system for maintaining the history of mechanical stimuli received (Fritton, J. McLeod \& Rubin, 2000; Turner et al., 2002; Moorer \& 
Stains, 2017).

Accumulated evidence has also suggested an additional important role of osteocytes in mobilizing minerals from the surrounding bone matrix via perilacunar/canalicular remodeling (Qing et al., 2012; Lotinun et al., 2019). Therefore, osteocytes may be the principal regulator for the functional bone adaptation (Skedros, Hunt \& Bloebaum, 2004; Hazenberg, Lee \& Taylor, 2006). Under culture conditions, osteocytes may retain information about their skeletal site of origin. For example, calvarial bone cells are much less sensitive to mechanical stimuli than ulnar bone cells (Rawlinson et al., 2009). Repetitive mechanical loads rapidly desensitize bone cells (Turner, 1998), indicating that bone tissue can be habituated, reducing its response to repetitive mechanical stimuli. Habituation is a term that describes the decrement in responsiveness to a repetitive stimulus in neuronal systems (McDiarmid, Yu \& Rankin, 2019).

Osteocytes play a crucial role in bone remodeling by controlling osteoblasts and osteoclasts via the expression of receptor activator of nuclear factor kappa B ligand (RANKL) and its decoy receptor, osteoprotegerin (OPG) (Nakashima et al., 2011; Xiong et al., 2011). Recent studies have shown that the main source of RANKL is osteocytes (Xiong et al., 2015). Correct osteoclastogenesis relies on a correct RANKL/OPG ratio (Capulli, Paone \& Rucci, 2014), and osteocytes express both factors at levels comparable with or exceeding those of osteoblasts (Bonewald, 2011).

Based on these findings, we hypothesized that osteocytes might be able to encode their loading history, with this encoded loading history regulating their gene expression even under in vitro culture. It has been stated that, in general, the disuse load was under 200 microstrains, the physiological load was between 200 and 2500 microstrains, and the overuse loading range was 2500-5000 microstrains when applying mechanical loads on the skeleton (Duncan \& Turner, 1995; 
93 Verbruggen, Vaughan \& McNamara, 2012). While, the osteocytes receive more mechanical force

94 since the findings suggested that osteocytes have mechanical signal amplification systems

95 (Takano-Yamamoto, 2014). The application of 2000 microstrains macroscopically to a piece of

96 bone resulted in a much greater microscopic strain surrounding the osteocyte lacunae of over

9730000 microstrains (Nicolella et al., 2006). A past study used finite element modeling analysis

98 which reported that global compressive loads of 150 microstrains (disuse), 1000 microstrains

99 (physiological), 3000 microstrains (overuse), and 5000 microstrains (pathological overload)

100 resulted in the maximum principal strains of $633,4272,12820$, and 21528 microstrains

101 respectively (Wang, Dong \& Xian, 2018). A previous study already measured the Young's

102 modulus of MLO-Y4 osteocyte-like cells as $1.98 \pm 0.25 \mathrm{kPa}$ and changed very slightly at a variant

103 indentation range $(500 \mathrm{~nm}-1000 \mathrm{~nm})$ by using atomic force microscopy (Wu et al., 2017).

104 Therefore, we applied approximate $3.1 \mathrm{~Pa}$ (resulted in $3.1 \mathrm{~Pa} / 1.98 \mathrm{kPa} \approx 1566$ microstrains) and

$1056.3 \mathrm{~Pa}$ (resulted in $6.3 \mathrm{~Pa} / 1.98 \mathrm{kPa} \approx 3182$ microstrains) compressive force to MLO-Y4 osteocyte-

106 like cells to simulate the bending loads-caused compressive stress in vivo within the pathological

107 load range.

108 In the present study, MLO-Y4 osteocyte-like cells were proliferated under a continuous 109 compressive force $\left(\approx 3.1 \mathrm{~Pa}\right.$ or 31 dynes $\left./ \mathrm{cm}^{2}\right)$ throughout the entire experiment after seeding the

110 cells $3 \mathrm{~h}$. A long-duration ( $36 \mathrm{~h}$ ) additional continuous compressive force (CCF) at $3.2 \mathrm{~Pa}$ was

111 applied as the treatment of loading history, followed by re-applying the same additional CCF $\approx \approx$

$1123.2 \mathrm{~Pa}$ or 32 dynes $\left./ \mathrm{cm}^{2}\right)$ for a short duration $(1 \mathrm{~h})$, to assess the effect of loading history on the

113 expression of interesting genes as well as the cell number, viability, morphology, and cell-to-cell

114 communications of MLO-Y4 osteocyte-like cells.

\section{Materials and Methods}




\section{Cell culture and reagents}

117 The MLO-Y4 cell line was purchased from Kerafast (Boston, MA, USA). MLO-Y4 cells (at 39

118 passages), an osteocyte-like cell line derived from the long bone of a transgenic female mouse

119 containing the osteocalcin promoter driving SV40 T-antigen (Kato et al., 1997), were seeded onto

120 the type I collagen-coated 24-well plates and cultured in alpha-modified Eagle's minimal essential

121 medium ( $\alpha$-MEM; Thermo Fisher Scientific, Waltham, MA, USA) containing 5.0\% heat-

122 inactivated fetal bovine serum (HIFBS; HyClone Laboratories, Logan, UT, USA), 5.0\% fetal calf

123 serum (FCS; HyClone), $100 \mathrm{U} / \mathrm{ml}$ penicillin, and $100 \mathrm{mg} / \mathrm{ml}$ streptomycin (Thermo Fisher

124 Scientific) at $37^{\circ} \mathrm{C}$ with $5 \% \mathrm{CO}_{2}$. MLO-Y4 cells express a high level of connexin $43(\mathrm{Cx} 43)$, early

125 osteocyte markers, such as podoplanin (Pdpn, also know at E11), Osteocalcin (OCN), and low

126 levels of some mature osteocyte markers, such as sclerostin (Sost) and dentin matrix protein 1

127 (Dmp1) (Yang et al., 2009; Dallas, Prideaux \& Bonewald, 2013; Sato et al., 2017).

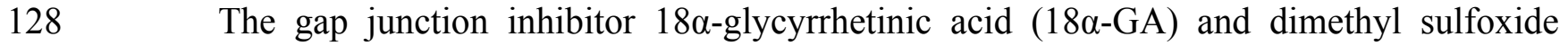
129 (DMSO) were purchased from Sigma-Aldrich (St. Louis, MO, USA). The following antibodies

130 were used: Rabbit anti-Cx43 polyclonal antibody (Cell Signaling, \#3512), Rabbit anti-phospho-

131 Cx43 (Ser368; pCx43) polyclonal antibody (Cell Signaling, \#3511), Goat anti-Sost polyclonal

132 antibody (R\&D system, AF1589), Mouse anti- $\beta$-actin monoclonal antibody (Sigma-Aldrich,

133 A5441), HRP-linked Goat anti-Rabbit IgG (Cell Signaling, \#7074S), HRP-linked Rabbit anti-Goat

134 IgG polyclonal antibody (R\&D system, HAF017), HRP-linked Goat anti-Mouse IgG polyclonal

135 antibody (Millipore, AP124P), Alexa Fluor ${ }^{\circledR} 594$ phalloidin (ThermoFisher, A12381), and Alexa

136 Fluor $^{\circledR} 488$ linked Goat anti-Rabbit polyclonal antibody (ThermoFisher, A11008) used for

137 Western blotting or immunofluorescence staining with MLO-Y4 osteocyte-like cells. 
139 The entire experiment design is shown in Figure 1. MLO-Y4 cells were seeded onto the type I

140 collagen-coated 24-well plate, 60-mm culture dish, or 35-mm glass-bottom (glass diameter: 14

$141 \mathrm{~mm}$ ) plastic dish at $2.63 \times 10^{4}$ cells $/ \mathrm{cm}^{2}$. Three hours after seeding the MLO-Y4 cells, a round

142 micro-cover glass (Fig. 1A; MATSUNAMI, Japan; area, $78.54 \mathrm{~mm}^{2}$; height, $0.21 \mathrm{~mm}$; diameter,10

$143 \mathrm{~mm}$; volume, $16.49 \mathrm{~mm}^{3}$; weight, $0.041 \mathrm{~g}$ ) or an assembly of two pieces of the normal cover glass

144 (Fig. 1A; MATSUNAMI, Japan; area, $960 \mathrm{~mm}^{2}$; height, $0.14 \mathrm{~mm}$; volume, $202.16 \mathrm{~mm}^{3}$; weight,

$1450.505 \mathrm{~g}$ ) was placed onto the MLO-Y4 cells as the background CCF of $31 \mathrm{dynes} / \mathrm{cm}^{2}$. As additional

146 CCF, a modified 200- $\mu 1$ pipette tip (Fig. 1A; density, $0.92 \mathrm{~g} / \mathrm{cm}^{3}$; height, $16 \mathrm{~mm}$; volume, 28.26

$147 \mathrm{~mm}^{3}$; weight, $0.211 \mathrm{~g}$ ) was placed onto the micro-cover glass. The modified $200-\mu 1$ pipette tip

148 itself generated 32 dynes $/ \mathrm{cm}^{2} \mathrm{CCF}$ when it was immersed into the well of the 24-well plate with

$1492.1 \mathrm{ml}$ culture media. Therefore, as shown in Figure 1, the MLO-Y4 cells in the loading history

150 group were grown under a total of $63 \mathrm{dynes} / \mathrm{cm}^{2} \mathrm{CCF}$, while the cells in the control group were

151 grown under only the background CCF (31 dynes $\left./ \mathrm{cm}^{2}\right)$.

152 After maintaining the MLO-Y4 cells under additional CCF for $36 \mathrm{~h}$, the additional CCF

153 was removed for $10 \mathrm{~h}$ (recovery period) before the same additional CCF was applied again for

154 another hour. One hour before applying the additional CCF again for an extra hour, $3.0 \mu \mathrm{M} 18 \alpha-$

155 GA or $0.1 \%$ DMSO was added and kept in the medium until the end of this experiment (Fig. 1B).

\section{Calcein-acetoxymethyl ester (Calcein-AM) and Hoechst 33342 staining}

157 During the $10-\mathrm{h}$ recovery period, the cell number and viability were examined at $0,3,6$, and $10 \mathrm{~h}$

158 using Calcein-AM and Hoechst 33342 staining. The micro-cover glasses were removed 15 minutes

159 prior to each time checkpoint, and the cells were loaded with $1.25 \mu \mathrm{M}$ Calcein-AM and $1 \mu \mathrm{g} / \mathrm{ml}$

160 Hoechst 33342 in the culture media for 15 minutes. Fluorescent and phase-contrast images of the

161 area that had been under the micro-cover glass were then taken with a KEYENCE BZ-9000 
162 fluorescence microscope (KEYENCE, Osaka, Japan) using a 20x phase-contrast lens.

\section{Cell numbers, viability, and morphological measurements}

164 After routine background correction using the ImageJ/Fiji software program (Schindelin et al., 165 2012), the background was neutralized using the "subtract background" (Sternberg, 1983) feature 166 of the ImageJ/Fiji tool. Finally, the number of cells with positive staining for Calcein-AM and 167 Hoechst 33342 and the cell morphological results were analyzed using the "Analyze Particles" 168 function after auto-thresholding with the default method in the ImageJ/Fiji tool.

169 Cell numbers were defined as the number of cell nuclei with positive staining on Hoechst

170 33342. The viable cell number was defined as the number of cells with positive staining on both

171 Calcein-AM or Hoechst 33342.

172 For the cell morphology, the aspect ratio, circularity, and solidity were measured. The 173 aspect ratio was defined as the ratio of the major axis to the minor axis of the best-fitted ellipse.

174 Circularity was defined as the $4 \pi \times$ cell area / perimeter ${ }^{2}$. Solidity was defined as the ratio of the 175 cell area to the convex area.

Reverse transcription and quantitative real-time polymerase chain reaction (qRT-PCR) synthesize complementary DNA (cDNA) with a ReverTra Ace qRT-PCR Kit (FSQ-201; Toyobo Co., Ltd., Osaka, Japan) in the total RNA concentration of $100 \mathrm{ng} / \mu 1$. The resulting cDNA products were diluted 5 times using pure water, and $1 \mu 1$ of 5 times diluted-cDNA product was used as a

181 template to quantify the relative content of messenger RNA (mRNA) by a qRT-PCR using SYBR ${ }^{\circledR}$

182 Green Real-time PCR Master Mix (QPK-201; Toyobo Co., Ltd., Osaka, Japan). The relative levels 183 of the PCR products were determined using a LightCycler System (Roche Diagnostics, Mannheim, 
184 Germany). The values of the threshold cycle $\left(\mathrm{C}_{\mathrm{t}}\right)$ were determined automatically by LightCycler

18596 software (version 1.1; Roche Diagnostics, Mannheim, Germany) with the default setting. The

186 sequences of the primers used in this study can be downloaded from Mendeley Data (http://dx.doi.

187 org/10.17632/2yfd2w8jfp.1\#file-c35071d0-dec9-47c0-84dc-351288a8356c). Differences in gene

188 expression levels following treatment were calculated using the $2^{-\Delta \Delta \mathrm{Ct}}$ method after normalization

189 within each sample of interesting gene expression levels against the expression levels of the 190 reference genes (Gapdh).

191 Fluorescence recovery after photobleaching (FRAP) assay and immunofluorescence staining

The MLO-Y4 osteocyte-like cells cultured in the glass-bottom dish were loaded with 1.25 $\mu \mathrm{M}$ Calcein-AM $1 \mathrm{~h}$ prior to the time point (45 h in Fig 1B) of re-applying CCF. The culture medium was refreshed two hours after loading with Calcein-AM, then the FRAP assay was performed alternately among the normal group (without cover glass and pipette tip), control group

196 (cultured with glass only), loading history group (cultured with glass and pipette tip after loading

197 history treatment), and CCF group (cultured with glass and pipette without loading history treatment) within 90 minutes.

FLUOVIEW FV500 confocal laser scanning microscopy (CLS) system (Olympus, Tokyo, Japan) equipped for differential interference contrast (DIC) microscopy. The CLS microscopy system was coupled to an inverted microscope (IX-71; Olympus) with a $\times 60$ (N.A. $=1.4)$ oil-

202 immersion objective lens. The scanning rate was $1.66 \mathrm{~s} / \mathrm{scan}$ for a 16 -bit image, $512 \times 512$ pixels in size. An MLO-Y4 cell surrounded by other cells under the cover glass (if applied), which was confirmed by the DIC view, was chosen for photobleaching. The boundary was enclosed and outlined with a rectangular region-of-interest tool. A predefined three-step FRAP procedure was automatically executed at a scan speed of $1.66 \mathrm{~s} / \mathrm{scan}$ on the largest cross section of the target cell. 
207 In the first step, a prebleached image of the whole field was taken using a low laser intensity $208($ AOTF $=10 \%$, zoom $=\times 1)$. The laser intensity was then increased $\times 100($ AOTF $=100 \%$, 209 zoom $=\times 40$ ), and the target cell was photobleached 1 time for about 2-3 s (depending on the area 210 of the target cell). Finally, the laser intensity and zoom were immediately reset to the prebleach 211 levels $(\mathrm{AOTF}=10 \%$, zoom $=\times 1)$, and time-lapse images were acquired with an interval of $15 \mathrm{~s}$.

212 The average intensity at each imaging time point was measured for three regions of interest:

213 the bleached target cell $\left(I_{t}\right)$, all other cells in the image field $\left(T_{t}\right)$ as control, and the non-fluorescent

214 region outside of all of the cells for background subtraction (BG) using the "Time Series Analyzer" 215 tool of ImageJ software (https://imagej.nih.gov/ij/plugins/time-series.html). The fluorescence 216 intensity of the target osteocyte $(F)$ was normalized as follows (Phair, Gorski \& Misteli, 2003):

$$
F_{t}=\frac{\left(T_{\text {prebleach }}-\mathrm{BG}\right)\left(I_{t}-\mathrm{BG}\right)}{\left(T_{t}-\mathrm{BG}\right)\left(I_{\text {prebleach }}-\mathrm{BG}\right)}
$$

The replacement of fluorescence within a bleached cell $\left(R_{t}\right)$ was calculated using the 218 following equation (Ishihara et al., 2008; Wang et al., 2016):

$$
R_{t}=\left[\left(F_{t}-F_{0}\right) /\left(F_{i}-F_{0}\right)\right] \times 100(\%)
$$

The percent replacement was defined as the fraction of molecules that were replaced during the time-course of the experiment. $F_{t}$ is the normalized fluorescence intensity at the time $(t)$ after

221 photobleaching by Eq. 1. $F_{0}$ is the normalized fluorescence immediately after photobleaching. $F_{i}$

222 is the initial fluorescence intensity before photobleaching.

Recovery curves could be analyzed for passive transport of fluorescent dyes through MLO-

224 Y4 osteocyte-like dendritic processes connected by gap junctions. Its kinetics follow the equation 225 (Wade, Trosko \& Schindler, 1986): 


$$
\frac{R_{p}-R_{t}}{R_{p}-R_{0}}=e^{-k t}
$$

226 where $R_{p}, R_{0}$, and $R_{t}$, are replacement of fluorescence in the bleached target cell at plateau

227 (equilibrium), zero time, and time ( $t$ ) after bleaching, respectively. The Eq. 3 could be rearranged

228 as $R_{t}=R_{0}+\left(R_{p}-R_{0}\right) \times\left(1-e^{-k t}\right)$, which is also called one-phase exponential association

229 equation. The parameter of $k$ and $R_{p}$ were estimated by a curve fit in Graphpad Prism software

230 (GraphPad Software, Inc., San Diego, CA, USA).

231 If we ignore the replacement of fluorescence during the photobleaching period, we could

232 take the $R_{p}$ as the mobile fraction $\left(\mathrm{f}_{\mathrm{m}}\right)$. The rate coefficient $\mathrm{k}$ is positively related to the permeability

233 coefficient of calcein.

234 To verify the protein expression of $\mathrm{Cx} 43$ and gap-junctional connections between MLO-

235 Y4 cells, an immunofluorescence staining was performed. MLO-Y4 osteocyte-like cells on a 236 glass-bottom dish were fixed with 4\% paraformaldehyde in phosphate-buffered saline (PBS) for $23710 \mathrm{~min}$, then permeabilized by incubation in $0.5 \%$ Triton X-100 in PBS for $10 \mathrm{~min}$. The cells were 238 blocked with Blocking One Histo (Nacalai tesque, Osaka, Japan) for 8 min at RT. After blocking, 239 the cells were incubated with Rabbit anti-Cx43 polyclonal antibody (overnight at $4{ }^{\circ} \mathrm{C}$ ), Alexa 240 Fluor $^{\circledR} 488$ linked Goat anti-Rabbit polyclonal antibody (1 h at RT), Alexa Fluor ${ }^{\circledR} 594$ phalloidin 241 (1 h at RT), and Hoechst 33342 (10 min at RT) in PBST containing 1\% bovine serum albumin 242 (BSA; Sigma-Aldrich, St. Louis, MO, USA). The cells were rinsed three times with PBST after 243 each above-mentioned step.

244 Western blot analyses

245 After the above-mentioned habituation experiment with CCF, the cells were washed with 246 cold PBS and lysed with a lysate buffer (1 mM Dithiothreitol, 1mM Phenylmethylsulfonyl 
247 Fluoride, $1 \mu \mathrm{g} / \mathrm{mL}$ leupeptin, $2 \mu \mathrm{g} / \mathrm{mL}$ aprotinin, 5mM EGTA) and sonicated on ice using a

248 supersonic machine. The protein concentration was determined using the Pierce ${ }^{\mathrm{TM}}$ BCA Protein

249 Assay Kit (Thermo Scientific, Waltham, MA, USA) and adjusted to a concentration of $1.1 \mathrm{mg} / \mathrm{ml}$

250 with the lysate buffer. Fifteen micrograms of each sample and Precision ${ }^{\mathrm{TM}}$ Plus Protein Dual Xtra

251 Standards markers (Bio-Rad, Berkeley, CA, USA) were separated by $7.5 \%$ or Any $\mathrm{kD}^{\mathrm{TM}}$

252 Criterion $^{\mathrm{TM}}$ TGX $^{\mathrm{TM}}$ Precast Gel (Bio-Rad, Berkeley, CA, USA) according to the predicted

253 molecular weight (MW) of our interesting proteins. The separated proteins were then transferred

254 to PVDF membranes (Millipore, Billerica, MA, USA). The membranes were blocked in a solution

255 consisting of 5\% nonfat skim milk in PBS containing 0.1\% Tween-20 (PBST) for $1 \mathrm{~h}$, and then

256 incubated $1 \mathrm{~h}$ at room temperature (RT) in Can Get Signal Immunoreaction Enhancer solution

257 (NKB-101; Toyobo Co., Ltd., Osaka, Japan) containing specific antibodies. The membranes were

258 washed 3 times (10 min each time) in PBST, then incubated for $1 \mathrm{~h}$ at RT with the corresponding

259 secondary antibodies. After washing 3 times, as described above, the proteins were detected by a

260 chemiluminescence system (ChemiDoc ${ }^{\mathrm{TM}}$ XRS+; Bio-Rad, Berkeley, CA, USA) with 20X

261 LumiGLO ${ }^{\circledR}$ Reagent and 20X Peroxide (Cell Signaling, Danvers, MA, USA). The densitometric

262 analysis of bands was performed using the ImageLab software (version 6.0.1; Bio-Rad, Berkeley,

263 CA, USA). The MW of our interesting proteins was estimated by a standard curve of the logarithm

264 of the MW versus relative migration distance that was generated using the Precision ${ }^{\mathrm{TM}}$ Plus Protein

265 Dual Xtra Standards.

266

267

268

269

\section{Statistical analyses}

All experiments in this study were performed in biological triplicated or quadruplicated wells or dishes with different batches. The cell that was cultured in 24-well plate within the first batch were used for morphological measurements and RNA extraction (see the assignment of treatment in 
270 supplementary Fig. 1). The cells cultured in $60 \mathrm{~mm}$ plastic dishes in the second batch were used

271 for protein extraction. The cells cultured in glass-bottom plastic dishes in the last batch were used

272 for FRAP experiments and immunofluorescence staining. The normality of all results was tested

273 by the Shapiro-Wilk test. A one-way analysis of variance (ANOVA) followed by a multiple

274 comparisons test with Fisher's least significant difference (LSD) test or with false discovery rate

275 (FDR) control was performed for the results with a normal distribution, while the Kruskal-Wallis

276 test followed by a multiple comparisons test with FDR control was performed for the results with

277 a non-normal distribution. The correlation of the expression of interesting genes with the cell

278 morphology (aspect ratio, circularity, and solidity) was examined by Pearson's correlation

279 coefficient and linear regression, but only significant correlations were plotted out in this study. A

280 two-way ANOVA followed by a multiple comparisons test with FDR control was applied to test

281 the differences of intracellular fluorescence intensity of calcein among different treatments at each

282 time checkpoint during the FRAP experiments.

283 All of the statistical analyses were performed using the GraphPad Prism software program, 284 version 8.0.0 for Windows (GraphPad Software, Inc., San Diego, CA, USA).

\section{Results}

MLO-Y4 cells maintained high viability under both 31 and 63 dynes $/ \mathrm{cm}^{2}$ for at least $36 \mathrm{~h}$

287 As Figure 2A and B show, the MLO-Y4 cells survived under both 31 and 63 dynes $/ \mathrm{cm}^{2} \mathrm{CCF}$ for 288 at least $36 \mathrm{~h}$. No significant differences in the cell number (239 cells $/ \mathrm{mm}^{2}$ on average in Fig. 3A)

289 or viability (93.67\% on average in Fig. 3B) under the micro-cover glass were observed between

290 the MLO-Y4 osteocytes with and without a loading history or among different time checkpoints 291 during the recovery period. 
292 The morphology changed during the recovery period

293 Figure 2C shows an example of the morphological measurements. At the end of this $10 \mathrm{~h}$ recover-

294 period, the aspect ratio was significantly increased only in the MLO-Y4 osteocytes without a 295 loading history (median aspect ratio of 1.7 vs. 2.0 with FDR value less than 0.001 in Fig. 4A). The 296 circularity of MLO-Y4 osteocytes with a loading history was lower than that of the cells without 297 a loading history immediately after removing the additional weight but ultimately returned to the 298 same level after the 10-h recovery period (Fig. 4B). The solidity (cell area / convex area) of the 299 MLO-Y4 osteocytes with a loading history was lower than that of the cells without a loading 300 history after the 10-h recovery period but the same level as when immediately removing the 301 additional weight (Fig. 4C). Similarly to the solidity, the cell area did not show any significant 302 differences between the MLO-Y4 cells under 63 dynes $/ \mathrm{cm}^{2}$ and 31 dynes $/ \mathrm{cm}^{2} \mathrm{CCF}$ for $36 \mathrm{~h}$, 303 although the cell area was significantly different after the 10-h recovery period (Fig. 4D).

304 The expression of most of the expression of our interesting genes recovered to the control level 305 after removal of the mechanical stimuli

306 The mRNA expression of RANKL, OPG, Gja1, and Pdpn was decreased, and the RANKL/OPG

307 ratio, Sost, Dmp1, and OCN higher in the MLO-Y4 osteocyte-like cells under 63 dynes $/ \mathrm{cm}^{2}$ than 308 in those under 31 dynes $/ \mathrm{cm}^{2}$ (Fig. 5). After changing the CCF from 63 dynes $/ \mathrm{cm}^{2}$ back to 31 309 dynes $/ \mathrm{cm}^{2}$ for $3 \mathrm{~h}$, the mRNA expression of RANKL, Gja1, Dmp1, Pdpn, and OCN recovered 310 back to the same level as in the MLO-Y4 osteocyte-like cells under 31 dynes $/ \mathrm{cm}^{2}$ (Figs. 5A, D, F, $311 \mathbf{G}$, and $\mathbf{H}$ ), but the mRNA expression of OPG increased, so the RANKL/OPG ratio consequently

312 decreased (Fig. 5C). Ultimately, the expression of RANKL and OPG and the RANKL/OPG ratio 313 returned to the same level as in the control group after changing the CCF from 63 dynes $/ \mathrm{cm}^{2}$ back 
314 to $31 \mathrm{dynes} / \mathrm{cm}^{2}$ for $10 \mathrm{~h}$ (Figs. 5A-C). However, the mRNA expression of Sost still higher in the

315 cells previously under 63 dynes $/ \mathrm{cm}^{2}$ than in those only under $31 \mathrm{dynes} / \mathrm{cm}^{2}$ even after changing

316 the CCF from 63 dynes/cm² back to 31 dynes $/ \mathrm{cm}^{2}$ for $10 \mathrm{~h}$ (Fig. 5E). Notably, the mRNA

317 expression of Dmp1 was suddenly increased in the cells previously under 63 dynes $/ \mathrm{cm}^{2}$ (with

318 loading history) at the end of this 10-h recovery period (Fig. 5F).

319 The expression profiles of OPG, Gjal, and Dmpl were significantly correlated with the cell 320 morphological changes during the recovery period

321 The mRNA expression profile of OPG, Gja1, and Dmp1 was significantly correlated with the

322 morphological changes in circularity, solidity, or area during the $10-\mathrm{h}$ recovery period (Figs. 5I-

323 L). Interestingly, more significant changes of all of the solidity (Fig. 4C), area (Fig. 4D), and the

324 mRNA expression profile of Dmpl (Fig. 5F) were observed at the end of the 10-h recovery period.

325 Please note that the Pearson's Correlation Coefficient was calculated using the mean value of both 326 morphological changes and mRNA expression fold change, but the linear regression test was 327 performed using the individual mRNA expression fold change against the mean value of 328 morphological changes.

329 The loading history reduced the responsiveness when the same CCF was applied again but could 330 be influenced by gap junctional inhibitor

331 The mRNA expression of RANKL, OPG, and the RANKL/OPG ratio in the MLO-Y4 osteocytes 332 without a loading history was dramatically changed (i vs. iii in Fig. 6A-C) in response to the new 333 1-h application of additional weight loading, but no significant changes were observed in the 334 MLO-Y4 osteocyte-like cells with a loading history (i vs. ii in Fig. 6A-C). However, the 335 expression of RANKL, OPG, and the RANKL/OPG ratio remained at the same level as in the 
336 control group in the MLO-Y4 osteocyte-like cells with a loading history (i vs. ii in Figs. 6A-C),

337 but similar findings were not found in the MLO-Y4 osteocyte-like cells treated with gap junction

338 inhibitor (v vs. viii in Figs. 6A-C). In other words, the mRNA expression of RANKL, OPG, and

339 the RANKL/OPG ratio was habituated by a 36-h CCF and was disrupted by junction inhibitor.

340 Notably, $18 \alpha$-GA treatment reduced the RANKL/OPG ratio by decreasing the RANKL

341 expression (iv vs. v in Figs. 6A and B). However, in response to the new application of 1-h loading

342 of additional weight in the MLO-Y4 osteocyte-like cells without a loading history (i vs. iii and $\mathbf{v}$

343 vs. vii in Figs. 6A-C), the mRNA expression of OPG showed a similar tendency to that in the

344 MLO-Y4 osteocytes with 18 $\alpha$-GA (comparing $\mathbf{i}$ vs. iii with $\mathbf{v}$ vs. vii in Figs. 6B), but the changes

345 in the MLO-Y4 osteocytes with 18 $\alpha$-GA were less significant than the MLO-Y4 osteocytes

346 without a loading history (comparing $\mathbf{i}$ vs. iii with $\mathbf{v}$ vs. vii in Figs. 6B). Interestingly, the increased

347 RANKL, the decreased OPG, and the increased RANKL/OPG ratio were observed in the 18a-GA-

348 treated MLO-Y4 osteocyte-like cells with a loading history in response to the re-application of 1-

$349 \mathrm{~h}$ loading of additional weight ( $\mathbf{v}$ vs. viii in Figs. 6A-C), a finding that was completely the opposite

350 of that in MLO-Y4 osteocyte-like cells without both 18a-GA treatment and loading

351 history(comparing $\mathbf{i}$ vs. iii with $\mathbf{v}$ vs. viii in Figs. 6A-C).

352 Whereas, the rest of our interesting genes (Figs. 6D-H) did not show such obvious

353 habituation phenomena or opposite trend between with and without 18a-GA in response to the

354 new 1-h application of additional weight loading as described above. However, the increase of

355 mRNA expression of Sost and OCN in the MLO-Y4 osteocyte-like cells with a loading history is

356 less than that in the MLO-Y4 osteocyte-like cells without a loading history (ii vs. iii in Figs. 6E

357 and $\mathbf{H}$ ). In other words, the mRNA expression of Sost and OCN showed sensitivity to the treatment

358 of loading history (ii vs. iii in Figs. 6E and $\mathbf{H}$ ). 
360 in our previous studies (Kamioka et al., 2007; Ishihara et al., 2008). However, the mode of

361 inhibitory action of GA is still not completely understood (Willebrords et al., 2017). Direct

362 interaction between $18 \alpha-G A$ and gap junctions (GJs) is possible when the former is inserted into

363 the plasma membrane, thereby binding to GJs and causing a conformational alteration

364 (Willebrords et al., 2017). Other possibilities include changes in the connexin phosphorylation

365 status, which led to a reduction in connexin expression (Willebrords et al., 2017). Our results (iv

366 vs. v in Fig. 6D, $P$-value $<0.05$ by unpaired $t$-test which did not show) also showed a reduction of

367 Gja1, which were in good agreement with the previous results (Willebrords et al., 2017), although

368 this reduction did not show significance by Fisher's LSD due to the big pooled variance.

Intercellular gap-junctional communications (GJIC) and Cx43 accumulation was reduced by

370 loading history

371

The GJIC among the MLO-Y4 osteocyte-like cells was confirmed by the

372 immunofluorescence staining of $\mathrm{Cx} 43$ and FRAP assay (Figs. 7A and B). Comparing with normal

373 cultured MLO-Y4 cells (i in Figs. 7C-G and 8), the MLO-Y4 cells cultured under glass (ii in Figs.

374 7C-G and 8) with (iii in Figs. 7C-G and 8) or without (iv in Figs. 7C-G and 8) loading history

375 showed decreased mobile fraction (i.e., lower recover percentage) in Figures 7 C and D with

376 decreased protein expression of Cx43 (Figs. 8A and B). Remarkably, the changes in the capacity

377 of GJIC and protein expression of Cx43 were opposite between the MLO-Y4 osteocyte-like cells

378 with and without treatment of loading history (comparing ii vs. iii with ii vs. iv in Figs. 7C, D, 8A,

379 and $\mathbf{B}$ ) in response to the application of 1-h loading of additional weight. Indeed, the significant

380 interaction effect was observed between mechanical stimuli (i.e., normal cultured, cultured under

381 cover glass with or without loading history) and time after photobleaching in Figure 7C, which 
382 suggested a significant influence of the loading history on the fluorescence recover curve. The

383 MLO-Y4 osteocyte-like cells without loading history showed a sharper recover curve and higher

384 coefficient $\mathrm{k}$ than that with loading history. In other words, the loading history disrupted the CCF-

385 caused GJIC increase. High $\mathrm{R}^{2}$ values shown in Figure 7F suggested a good fitness of the actual

386 recover curve to the theoretically predicted curve (Fig. 7C). No significant differences were

387 observed in the number of neighboring cells, which implied that the GJIC differences among

388 groups in this study were not caused by the different number of neighboring cells.

389 The ratio of $\mathrm{pCx} 43$ to total $\mathrm{Cx} 43$ was unchanged among the different treatment of

390 mechanical stimuli (Fig. 8A and D). Although the mRNA expression of Sost showed sensitivity

391 to the treatment of loading history (ii vs. iii in Fig. 6E), the protein expression of Sost did not show

392 significant differences between the MLO-Y4 osteocyte-like cells with and without loading history

393 (ii vs. iii in Figs. 8A and $\mathbf{E}$ ).

\section{Discussion}

395 In this study, the phenotype of MLO-Y4 cells was confirmed by checking the mRNA expression

396 of Pdpn, Cx43, RANKL, OCN, Dmp1, Sost, and OPG, respectively, with the average $C_{t}$ values of

$39718.13,19.75,22.22,23.74,28.93,30.74$, and 33.92 comparing to the average $C_{t}=14.03$ of Gapdh

398 (raw $\mathrm{C}_{\mathrm{t}}$ values could be downloaded from Mendeley Data:

399 http://dx.doi.org/10.17632/2yfd2w8jfp.1\#file-22aa2376-6feb-4709-b37e-dfdc8c8cf309). The

400 protein expression of $\mathrm{Cx} 43$ and Sost was confirmed by Western blot (Fig. 8A). The GJIC was

401 confirmed by the FRAP assay and immunofluorescence staining of Cx43 (Fig. 7A and B). The

402 dendritic cellular processes were also observed (Figs. 2, 7A, and B). Therefore, the MLO-Y4 cells

403 used in this study were characterized as the osteocyte-like cells.

404 Our results showed that MLO-Y4 cells maintained high viability under both 31 and 63 
405 dynes $/ \mathrm{cm}^{2}$ for at least $36 \mathrm{~h}$ (Fig. 3B); however, the proliferation may be inhibited. The grown area 406 covering each well of the 24 -well plate was $1.9 \mathrm{~cm}^{2}$, so the estimated cell number in total was 40745,410 cells/well, based on our results shown in Figure 3A (239 cells $\left./ \mathrm{mm}^{2}\right)$, which was even 408 smaller than our seeding density $(50,000$ cells/well $)$. The difference in the density between the 409 MLO-Y4 cells grown under a cover glass and without a cover glass was very obvious at the edge 410 of the cover glass (supplementary Fig. 2). This may be because placing the cover glass directly 411 on the cell may have created a relatively sealed environment that reduced nutrition. This relatively 412 sealed environment is similar to the environment of mineralized tissue. Moreover, this condition 413 also reduced the proliferation of MLO-Y4 osteocytes that may impact the cell morphology and 414 gene expression profile. A previous microarray study showed that the MLO-Y4 osteocytes had a 415 different gene expression profile between conditions of low and high cell density (Yang et al., 416 2009). Cell density may also influence cell morphology. A relatively sealed environment

417 combined with the inhibition of proliferation ultimately created an ideal condition for us to observe 418 the cell morphological changes during the 10 -h recovery period after $36-\mathrm{h}$ additional CCF 419 exposure.

420 In the current study, we developed an in vitro platform mimicking the habituation 421 phenomena observed in in vivo or ex vivo bone tissues that provide a more economic and 422 convenient approach to investigating the possible mechanism of encoding the loading history in 423 osteocytes. With this platform, all MLO-Y4 osteocytes were grown under background CCF, which 424 is similar to the in vivo conditions, as all osteocytes in our bodies are growing under a complicated 425 loading environment (Turner, 1998). Unlike to hydrostatic pressure, compressive pressure 426 generates the uniaxial deformation that is also an important mechanical type received by osteocytes 427 in vivo by daily activities (Duncan \& Turner, 1995). 
429 The reduced responsiveness of the RANKL/OPG expression to the re-application of the same CCF

430 was diminished in the MLO-Y4 osteocytes with a loading history, suggesting that the in vitro

431 cultured MLO-Y4 osteocytes could be habituated by 36-h CCF application in a loading 432 environment with background CCF (comparing $\mathbf{i}$ vs. iii with $\mathbf{i}$ vs. ii in Figs. 5A-C and 6A-C).

433 Loading history significantly suppressed the CCF-induced increase of mRNA expression of Sost

434 and OCN (ii vs. iii in Figs. 6E and $\mathbf{H}$ ). However, no significant differences in protein expression 435 changes (iii vs. iv in Figs. 8A and E) in Sost between the MLO-Y4 osteocyte-like cells with and 436 without loading history in response to the application of the additional loading weight for $1 \mathrm{~h}$.

437 Treatment of loading history reversed the response of the protein expression of Cx43 and GJIC to 438 the re-application of the same CCF for $1 \mathrm{~h}$ (comparing ii vs. iii with ii vs. iv in Figs. 7C-D and 439 8A-B). However, the treatment of loading history did not show any significant influences on the 440 mRNA expression of Cx43 (ii vs. iii in Fig. 6D).

441 It is well documented that mechanical stimuli changed the protein secretion in many other 442 types of cells (Apodaca, 2002). Since the Sost is a secreted protein (Poole et al., 2005), the 443 discrepancy between the mRNA and protein expression of Sost may be due to the mechanical 444 stimuli-induced changes in protein secretion. Indeed, our previous study found that mechanical 445 unloading changed the concentration of extracellular Sost but not the intracellular Sost in the femur 446 in vivo using a transmission electron microscopy (Osumi et al., 2020). On the other hand, the 447 discrepancy between the mRNA and protein expression of $\mathrm{Cx} 43$ may be due to the post448 translational modifications, since the mechanical stimulation changed the phosphorylation status 449 of Cx43 (Genetos et al., 2007; Qin et al., 2020) and Cx43 degradation is controlled by complex 450 crosstalk between connexin phosphorylation and ubiquitination (Totland et al., 2020). Ser368 of 
451 Cx43 is phosphorylated by protein kinase C (PKC), which decreases cell-to-cell communication

452 (Lampe et al., 2000). However, the $\mathrm{pCx} 43 / \mathrm{Cx} 43$ ratio in the Figures $\mathbf{8 A}$ and $\mathbf{D}$ did not show

453 significant differences among all groups, which indicated that the loading history-induced changes

454 in GJIC and protein expression of $\mathrm{Cx} 43$ in this study was not due to the phosphorylation of Ser368

455 of Cx43 by PKC. Mitogen-activated protein kinase (MAPK)-mediated phosphorylation of Cx43

456 at serine residues 255, 262, 279, and 282 was related to the Cx43 degradation (Totland et al., 2020).

457 Lots of studies showed that the MAPK pathway could be rapidly activated by various cellular

458 mechanical stimuli (Takano-Yamamoto, 2014). Therefore, it is worthy of further studies in the

459 future which the role of the MAPK pathway in the loading history mediated gene expression

460 revealed by this study. The above discussions indicated that loading history might also have an

461 influence on the post-translational modifications on proteins, which is not fully supported by this

462 study but worthy of further investigations.

463 Blockade of junctions influenced the habituation effect

464 Gap junctions are hexametric channels formed by two docked hemichannels from adjacent cells

465 that permit the direct intercellular transfer of small signaling molecules, such as inositol

466 phosphates, cyclic nucleotides, and ATP. Connexins are the main components of gap junctions

467 and hemichannels, and the most abundant connexin in bone cells is Cx43 (Ishihara et al., 2008;

468 Manuscript, Plotkin \& Bellido, 2013), which is highly expressed in MLO-Y4 cells (Kato et al.,

469 1997). Cx43 is a key component of intracellular machinery responsible for signal transduction in

470 bone in response to variant stimuli (Manuscript, Plotkin \& Bellido, 2013). Our results showed that

471 18 $\alpha$-GA treatment of MLO-Y4 osteocytes with a loading history rescued their sensitivity to

472 RANKL and OPG expression in response to the re-application of CCF (comparing $\mathbf{i}$ vs. ii with $\mathbf{v}$ 473 vs. viii in Figs. 6A-C). 
475 a similar condition to the conditional deletion of $\mathrm{Cx} 43$ in mice, since osteocytes in vivo are always 476 under a complicated loading environment. Therefore, the conditional deletion of $\mathrm{Cx} 43$ in mice is

477 likely to block the gap junction in MLO-Y4 osteocytes with a certain loading history but not in 478 those without any history of mechanical stimuli exposure. Therefore, our in vitro findings are 479 consistent with those of previous reports in vivo.

480 A previous study showed a greater response to loading at the endocortical surface than the 481 periosteal surface (Birkhold et al., 2016). However, the finite elements analysis in this above482 mentioned study (Birkhold et al., 2016) showed that the endocortical surface was less strained than 483 the periosteal surface. Our findings may provide a possible explanation for this observation that 484 osteocytes under higher-magnitude loading may be more habituated than those under lower485 magnitude loading, thereby showing less sensitivity to mechanical stimuli. In addition, we found 486 that the expression of our interesting genes in MLO-Y4 osteocytes under $31 \mathrm{dynes} / \mathrm{cm}^{2} \mathrm{CCF}$ was 487 more sensitive to additional CCF than MLO-Y4 osteocytes that had previously been exposed to 488 63 dynes/cm² CCF (Figs. 6 and 8 ).

A number of previous studies have reported an enhanced response to mechanical 490 stimulation in mice with conditional deletion of $\mathrm{Cx} 43$ from either osteoblasts or osteocytes (Zhang 491 et al., 2011), osteochondroprogenitors (Grimston et al., 2012), and osteocytes only (Bivi et al., 492 2013). These reports supported our present finding that $18 \alpha-G A$ treatment increased the sensitivity 493 to CCF in the MLO-Y4 osteocytes with a loading history (Fig. 6). Deletion of Cx43 in mature 494 osteoblasts and osteocytes (Plotkin et al., 2008) or in osteocytes only (Bivi et al., 2012) does not 495 decrease the bone mass. Quite the contrary, it increases the periosteal bone formation in the pattern 496 of a bone subjected to modeling during growth (Bivi et al., 2012), which is in agreement with our 
497 results that blockade of the gap junction by $18 \alpha-\mathrm{GA}$ decreased the RANKL/OPG ratio.

498 Given the above, our findings suggest that gap junctional intercellular communications

499 may be involved in the habituation of osteocytes.

500 The mRNA expression of OPG, Gjal, and Dmpl showed significant correlation to cell 501 morphological changes during the recovery period

502 Intriguingly, the expression profile of OPG, Gja1, and Dmp1 showed a significant correlation with 503 the morphological changes in the MLO-Y4 osteocytes with a loading history (Figs. 5I-L). $504 \mathrm{Gja1} / \mathrm{Cx} 43$ has been reported to control cell morphology and migration in other types of cells (Xu 505 et al., 2006; Liu et al., 2012; Machtaler et al., 2014). However, the authors do not find the previous 506 studies reported the correlations between cell morphological changes and the mRNA expression 507 of OPG or Dmp1.

$508 \quad$ Notably, both the solidity and cell area of the MLO-Y4 osteocytes with a loading history 509 were significantly different from in those without a loading history after the 10-h recovery period, 510 even it was at the same level at the beginning of the recovery period (Fig. 4C and D). Similarly, 511 more significant changes of all of the mRNA expression profile of Dmp1 (Fig. 5F) were observed 512 at the end of the 10-h recovery period, which is significantly correlated to the solidity and cell area

513 (Figs. 5K and $\mathbf{L}$ ). According to the definition of the solidity in the ImageJ/Fiji software program

514 (Schindelin et al., 2012), a lower solidity may suggest more cellular dendritic processes in MLO-

515 Y4 osteocytes since the expended slim dendritic processes would greatly increase the convex area

516 but contribute slightly to the entire cell area (Fig. 2C). It was also demonstrated that fluid shear

517 stress changed the cell morphology of osteoblast-like IDG-SW3 cells (Xu et al., 2018), and 48 h

518 of clinorotation changed the $\alpha$-tubulin distribution in MLO-Y4 cells (Xu et al., 2012). Indeed, our

519 results showed that the cell morphology was not only immediately changed after the mechanical 
520 stimuli exposure but also gradually changed over $10 \mathrm{~h}$ after a long period of CCF loading (Fig. 4).

521 A previous study from our group showed that mechanical loading induced morphological

522 changes in osteocytes in vivo (Sugawara et al., 2013). Recent studies showed that the cell

523 morphological changes determine the direction of human mesenchymal stem cell (hMSC)

524 differentiation through activating or inhibiting differential biological signals (Fan et al., 2019). For

525 example, hMSCs with a spread shape will differentiate to osteoblasts, those with a round shape

526 will differentiate to adipocytes, etc. Actin, one of the three major components of the cytoskeleton,

527 regulates the cell shape by controlling the dynamic equilibrium between the monomeric and

528 filamentous states, which also presents in the nucleus for transcriptional regulation (Misu,

529 Takebayashi \& Miyamoto, 2017). There are numerous studies showing that morphological

530 differences in osteocytes always correlate to some changes in gene expression or metabolomics

531 (Bacabac et al., 2008; Sasaki et al., 2012; Xu et al., 2012); however, the underlying mechanism

532 remains unclear and should be examined in future studies.

533 Given the discussion above, our findings suggest that CCF-induced cell morphological

534 changes may be involved in the habituation of osteocytes.

535

536

537

538

539

540

541 from all our results. For example, since the linear regression test was performed using the

542 individual mRNA expression fold change against the mean value of morphological changes, the 
543 discrepancy between $P$-values for Pearson's correlation coefficient and non-zero slope in Figure

544 5J suggested a high intraclass variance, which was also suggested by the long error bars in Figures

545 5A-H and 6.

546 Summary

547 Our findings showed that the mRNA expression of RANKL, OPG, Sost, and OCN in

548 MLO-Y4 osteocyte-like cells with treatment of loading history showed the decrement in

549 responsiveness to a re-application of the same CCF as the loading history (Fig. 6). This habituation

550 phenomenon could be disrupted by a GJIC inhibitor, which suggested the MLO-Y4 osteocyte-like

551 cells network is important for this habituation in vitro (Fig. 6). This behavior of in vitro cultured

552 MLO-Y4 osteocyte-like cells network is pretty similar to that of mathematical modeling of small

553 world networks (Strogatz, 2001) that was used to explain how rest-inserted loading arises more

554 bone formation than continuous cyclic loading (Gross et al., 2004). A small world network exhibit

555 power law behavior, in which small magnitude events occur often, whereas large magnitude events

556 occur infrequently (Latora \& Marchiori, 2001; Strogatz, 2001; Gross et al., 2004). A small world

557 network also exhibits threshold behavior (Latora \& Marchiori, 2001; Strogatz, 2001; Gross et al.,

558 2004). The decreased GJIC by the treatment of loading history (Fig. 7) blocked the connections

559 between the MLO-Y4 osteocyte-like cells then consequently decreased the efficient of the small

560 world network since the decreased connection range $(k)$ and probability $(p)$ in this small world

561 mathematic model (Latora \& Marchiori, 2001). Therefore, the raised threshold of the small word

562 network by efficient changes may be a possible mechanism to explain the habituation behavior of

563 in vitro cultured MLO-Y4 osteocyte-like cells observed in this study. However, this hypothesis

564 needs to be further confirmed by more supportive evidence in the future. Since the osteocytes

565 network is important for bone adaptation (Robling, 2012), findings about the behavior of in vitro 
566 cultured MLO-Y4 osteocyte-like cells network in response to loading history from this study may

567 have contributions to the further understanding in the molecular mechanism of the bone adaptation

568 and finally may contribute to reduce the risk of bone fracture by mediation of bone adaptation.

569

570

571

572

573

574

575

576

577

578

579

580

581

582

583

584

585

586

587

588

\section{Author contributions}

Ziyi Wang: Conceptualization, Visualization. Ziyi Wang and Yao Weng: Investigation, Data curation, Formal analysis, Writing_-Original Draft. Ziyi Wang, Yoshihito Ishihara, Naoya Odagaki, and Ei Ei Hsu Hlaing: Methodology. Ziyi Wang, Takashi Izawa, and Hiroshi Kamioka Funding acquisition, Resources. Ziyi Wang, Yao Weng, Yoshihito Ishihara, Naoya Odagaki, Ei Ei Hsu Hlaing, Takashi Izawa, Hirohiko Okamura, and Hiroshi Kamioka: Writing-Reviewing and Editing. Hirohiko Okamura, and Hiroshi Kamioka: Supervision.

\section{Acknowledgments}

The present work was supported by a Grant-in-Aid for Scientific Research (to Ziyi Wang [19J11906], Takashi Izawa [18H03011, 18KK0464], and Hiroshi Kamioka [19H03859]) from the Japan Society for the Promotion of Science, Japan. Ziyi Wang would also like to thank Shanqi Fu and Dr. Takako Hattori of the Department of Biochemistry and Molecular Dentistry of Okayama University Graduate School of Medicine Dentistry and Pharmaceutical Sciences for providing valuable advice.

\section{References}

Apodaca G. 2002. Modulation of membrane traffic by mechanical stimuli. American Journal of Physiology-Renal Physiology 282:F179-F190. DOI: 10.1152/ajprenal.2002.282.2.F179.

Bacabac RG, Mizuno D, Schmidt CF, MacKintosh FC, Van Loon JJWA, Klein-Nulend J, Smit TH. 2008. Round versus flat: Bone cell morphology, elasticity, and mechanosensing. Journal of Biomechanics 41:1590-1598. DOI: 10.1016/j.jbiomech.2008.01.031. 
589 Birkhold AI, Razi H, Duda GN, Weinkamer R, Checa S, Willie BM. 2016. The Periosteal Bone $590 \quad$ Surface is Less Mechano-Responsive than the Endocortical. Scientific Reports 6:23480. DOI: $591 \quad 10.1038 / \operatorname{srep} 23480$.

592 Bivi N, Condon KW, Allen MR, Farlow N, Passeri G, Brun LR, Rhee Y, Bellido T, Plotkin LI. 593 2012. Cell autonomous requirement of connexin 43 for osteocyte survival: Consequences for 594 endocortical resorption and periosteal bone formation. Journal of Bone and Mineral Research 595 27:374-389. DOI: 10.1002/jbmr.548.

Bivi N, Pacheco-Costa R, Brun LR, Murphy TR, Farlow NR, Robling AG, Bellido T, Plotkin LI. 2013. Absence of Cx43 selectively from osteocytes enhances responsiveness to mechanical force in mice. Journal of Orthopaedic Research 31:1075-1081. DOI: 10.1002/jor.22341.

Bonewald LF. 2011. The amazing osteocyte. Journal of Bone and Mineral Research 26:229-238. DOI: $10.1002 / \mathrm{jbmr.320.}$

Capulli M, Paone R, Rucci N. 2014. Osteoblast and osteocyte: Games without frontiers. Archives 602 of Biochemistry and Biophysics 561:3-12. DOI: 10.1016/j.abb.2014.05.003.

Dallas SL, Prideaux M, Bonewald LF. 2013. The Osteocyte: An Endocrine Cell ... and More. Endocrine Reviews 34:658-690. DOI: 10.1210/er.2012-1026.

Duncan RL, Turner CH. 1995. Mechanotransduction and the functional response of bone to 606 mechanical strain. Calcified Tissue International 57:344-358. DOI: 10.1007/BF00302070.

607 Fan Y-L, Zhao H-C, Li B, Zhao Z-L, Feng X-Q. 2019. Mechanical Roles of F-Actin in the 608 609 Differentiation of Stem Cells: A Review. ACS Biomaterials Science \& Engineering. DOI: 10.1021/acsbiomaterials.9b00126.

610 Fritton SP, J. McLeod K, Rubin CT. 2000. Quantifying the strain history of bone: Spatial 611 uniformity and self-similarity of low-magnitude strains. Journal of Biomechanics 33:317- 
325. DOI: 10.1016/S0021-9290(99)00210-9.

613 Genetos DC, Kephart CJ, Zhang Y, Yellowley CE, Donahue HJ. 2007. Oscillating fluid flow 614 activation of gap junction hemichannels induces atp release from MLO-Y4 osteocytes. 615 Journal of Cellular Physiology 212:207-214. DOI: 10.1002/jcp.21021.

616 Grimston SK, Watkins MP, Brodt MD, Silva MJ, Civitelli R. 2012. Enhanced periosteal and 617 endocortical responses to axial tibial compression loading in conditional connexin43 deficient 618 mice. PLoS ONE 7:1-8. DOI: 10.1371/journal.pone.0044222.

619 Gross TS, Poliachik SL, Ausk BJ, Sanford DA, Becker BA, Srinivasan S. 2004. Why Rest 620 Stimulates Bone Formation: A Hypothesis Based on Complex Adaptive Phenomenon. 621 Exercise and Sport Sciences Reviews 32:9-13. DOI: 10.1097/00003677-200401000-00003.

622 Hazenberg JG, Lee TC, Taylor D. 2006. The role of osteocytes in functional bone adaptation. 623 BoneKEy-Osteovision 3:10-16. DOI: 10.1138/20060198.

624 Ishihara Y, Kamioka H, Honjo T, Ueda H, Takano-Yamamoto T, Yamashiro T. 2008. Hormonal, $625 \mathrm{pH}$, and calcium regulation of connexin 43-mediated dye transfer in osteocytes in chick 626 calvaria. Journal of bone and mineral research : the official journal of the American Society 627 for Bone and Mineral Research 23:350-60. DOI: 10.1359/jbmr.071102.

628 Kamioka H, Ishihara Y, Ris H, Murshid SA, Sugawara Y, Takano-Yamamoto T, Lim SS. 2007. 629 Primary cultures of chick osteocytes retain functional gap junctions between osteocytes and 630 between osteocytes and osteoblasts. Microscopy and Microanalysis 13:108-117. DOI: 10.1017/S143192760707016X.

632 Kamioka H, Kameo Y, Imai Y, Bakker AD, Bacabac RG, Yamada N, Takaoka A, Yamashiro T, 633 Adachi T, Klein-Nulend J. 2012. Microscale fluid flow analysis in a human osteocyte 634 canaliculus using a realistic high-resolution image-based three-dimensional model. 
Integrative Biology 4:1198-1206. DOI: 10.1039/c2ib20092a.

636

637

638

639

640

641

642

643

644

645

646

647

648

649

650

651

652

653

654

655

656

657

Kato Y, Windle JJ, Koop BA, Mundy GR, Bonewald LF. 1997. Establishment of an Osteocytelike Cell Line, MLO-Y4. Journal of Bone and Mineral Research 12:2014-2023. DOI: 10.1359/jbmr.1997.12.12.2014.

Kerschnitzki M, Kollmannsberger P, Burghammer M, Duda GN, Weinkamer R, Wagermaier W, Fratzl P. 2013. Architecture of the osteocyte network correlates with bone material quality. Journal of Bone and Mineral Research 28:1837-1845. DOI: 10.1002/jbmr.1927.

Kriegman S, Blackiston D, Levin M, Bongard J. 2020. A scalable pipeline for designing reconfigurable organisms. Proceedings of the National Academy of Sciences:201910837. DOI: $10.1073 /$ pnas.1910837117.

Lampe PD, TenBroek EM, Burt JM, Kurata WE, Johnson RG, Lau AF. 2000. Phosphorylation of Connexin43 on Serine368 by Protein Kinase C Regulates Gap Junctional Communication. Journal of Cell Biology 149:1503-1512. DOI: 10.1083/jcb.149.7.1503.

Latora V, Marchiori M. 2001. Efficient behavior of small-world networks. Physical Review Letters 87:198701-1-198701-4. DOI: 10.1103/PhysRevLett.87.198701.

Liu X, Sun L, Torii M, Rakic P. 2012. Connexin 43 controls the multipolar phase of neuronal migration to the cerebral cortex. Proceedings of the National Academy of Sciences 109:82808285. DOI: $10.1073 /$ pnas. 1205880109.

Lotinun S, Ishihara Y, Nagano K, Kiviranta R, Carpentier V, Neff L, Parkman V, Ide N, Hu D, Dann P, Brooks D, Bouxsein ML, Wysolmerski J, Gori F, Baron R. 2019. Cathepsin Kdeficient osteocytes prevent lactation-induced bone loss and parathyroid hormone suppression. Journal of Clinical Investigation 25:3058-3071. DOI: 10.1172/jci122936.

Machtaler S, Choi K, Dang-Lawson M, Falk L, Pournia F, Naus CC, Matsuuchi L. 2014. The role 
658

659

660

661

662

663

664

665

666

667

668

669

670

671

672

673

674

675

676

677

678

679

680

of the gap junction protein connexin43 in B lymphocyte motility and migration. FEBS Letters 588:1249-1258. DOI: 10.1016/j.febslet.2014.01.027.

Manuscript A, Plotkin LI, Bellido T. 2013. Beyond gap junctions: Connexin43 and bone cell signaling. Bone 52:157-166. DOI: 10.1016/j.bone.2012.09.030.

McDiarmid TA, Yu AJ, Rankin CH. 2019. Habituation Is More Than Learning to Ignore: Multiple Mechanisms Serve to Facilitate Shifts in Behavioral Strategy. BioEssays 41:1900077. DOI:

10.1002/bies.201900077.

Misu S, Takebayashi M, Miyamoto K. 2017. Nuclear actin in development and transcriptional reprogramming. Frontiers in Genetics 8:1-8. DOI: 10.3389/fgene.2017.00027.

Moorer MC, Stains JP. 2017. Connexin43 and the Intercellular Signaling Network Regulating Skeletal Remodeling. Current Osteoporosis Reports 15:24-31. DOI: 10.1007/s11914-0170345-4.

Nakashima T, Hayashi M, Fukunaga T, Kurata K, Oh-Hora M, Feng JQ, Bonewald LF, Kodama T, Wutz A, Wagner EF, Penninger JM, Takayanagi H. 2011. Evidence for osteocyte regulation of bone homeostasis through RANKL expression. Nat Med 17:1231-1234. DOI: $10.1038 / \mathrm{nm} .2452$.

Nicolella DP, Moravits DE, Gale AM, Bonewald LF, Lankford J. 2006. Osteocyte lacunae tissue strain in cortical bone. Journal of Biomechanics 39:1735-1743. DOI: 10.1016/j.jbiomech.2005.04.032.

Osumi R, Wang Z, Ishihara Y, Odagaki N, Iimura T, Kamioka H. 2020. Changes in the intra- and peri-cellular sclerostin distribution in lacuno-canalicular system induced by mechanical unloading. Journal of Bone and Mineral Metabolism. DOI: 10.1007/s00774-020-01135-9.

Phair RD, Gorski SA, Misteli T. 2003. Measurement of Dynamic Protein Binding to Chromatin 
In Vivo, Using Photobleaching Microscopy. In: Methods of Enzimology. 393-414. DOI:

682 10.1016/S0076-6879(03)75025-3.

683 Plotkin LI, Lezcano V, Thostenson J, Weinstein RS, Manolagas SC, Bellido T. 2008. Connexin 68443 is required for the anti-apoptotic effect of bisphosphonates on osteocytes and osteoblasts 685 in vivo. Journal of Bone and Mineral Research 23:1712-1721. DOI: 10.1359/jbmr.080617.

686 Poole KES, Van Bezooijen RL, Loveridge N, Hamersma H, Papapoulos SE, Löwik CW, Reeve J. 687 2005. Sclerostin is a delayed secreted product of osteocytes that inhibits bone formation. The 688 FASEB Journal 19:1842-1844. DOI: 10.1096/fj.05-4221 fje.

689 Qin L, Liu W, Cao H, Xiao G. 2020. Molecular mechanosensors in osteocytes. Bone Research 690 8:1-24. DOI: 10.1038/s41413-020-0099-y.

691 Qing H, Ardeshirpour L, Divieti Pajevic P, Dusevich V, Jähn K, Kato S, Wysolmerski J, Bonewald 692 LF. 2012. Demonstration of osteocytic perilacunar/canalicular remodeling in mice during 693 lactation. Journal of Bone and Mineral Research 27:1018-1029. DOI: 10.1002/jbmr.1567.

694 Rawlinson SCF, Mosley JR, Suswillo RFL, Pitsillides AA, Lanyon LE. 2009. Calvarial and limb 695 bone cells in organ and monolayer culture do not show the same early responses to dynamic 696 mechanical strain. Journal of Bone and Mineral Research 10:1225-1232. DOI: 697 $10.1002 / \mathrm{jbmr} .5650100813$.

698 Robling AG. 2012. The interaction of biological factors with mechanical signals in bone 699 adaptation: Recent developments. Current Osteoporosis Reports 10:126-131. DOI: 700

Sasaki M, Hongo H, Hasegawa T, Suzuki R, Zhusheng L, de Freitas PHL, Yamada T, Oda K, 702 703 Yamamoto T, Li M, Totsuka Y, Amizuka N. 2012. Morphological aspects of the biological function of the osteocytic lacunar canalicular system and of osteocyte-derived factors. Oral 
705 Sato M, Suzuki T, Kawano M, Tamura M. 2017. Circulating osteocyte-derived exosomes contain 706 miRNAs which are enriched in exosomes from MLO-Y4 cells. Biomedical Reports 6:223231. DOI: $10.3892 / \mathrm{br} .2016 .824$.

Schindelin J, Arganda-Carreras I, Frise E, Kaynig V, Longair M, Pietzsch T, Preibisch S, Rueden C, Saalfeld S, Schmid B, Tinevez J-Y, White DJ, Hartenstein V, Eliceiri K, Tomancak P, Cardona A. 2012. Fiji: an open-source platform for biological-image analysis. Nature Methods 9:676-682. DOI: 10.1038/nmeth.2019.

712

Skedros JG, Hunt KJ, Bloebaum RD. 2004. Relationships of loading history and structural and material characteristics of bone: Development of the mule deer calcaneus. Journal of Morphology 259:281-307. DOI: 10.1002/jmor.10167.

715 Sternberg. 1983. Biomedical Image Processing. Computer 16:22-34. DOI: 10.1109/MC.1983.1654163.

717 Strogatz SH. 2001. Exploring complex networks. Nature 410:268-276. DOI: 10.1038/35065725.

718 Sugawara Y, Kamioka H, Ishihara Y, Fujisawa N, Kawanabe N, Yamashiro T. 2013. The early 719 mouse 3D osteocyte network in the presence and absence of mechanical loading. Bone 720

Takano-Yamamoto T. 2014. Osteocyte function under compressive mechanical force. Japanese 52:189-196. DOI: 10.1016/j.bone.2012.09.033.

Totland MZ, Rasmussen NL, Knudsen LM, Leithe E. 2020. Regulation of gap junction 724 intercellular communication by connexin ubiquitination: physiological and 725 pathophysiological implications. Cellular and Molecular Life Sciences 77:573-591. DOI: 10.1007/s00018-019-03285-0. 
727 Turner CH. 1998. Three rules for bone adaptation to mechanical stimuli. Bone 23:399-407. DOI:

$728 \quad 10.1016 / \mathrm{S} 8756-3282(98) 00118-5$.

729 Turner CH, Pavalko FM. 1998. Mechanotransduction and functional response of the skeleton to

730 physical stress: The mechanisms and mechanics of bone adaptation. Journal of Orthopaedic

$731 \quad$ Science 3:346-355. DOI: 10.1007/s007760050064.

732 Turner CH, Robling AG, Duncan RL, Burr DB. 2002. Do Bone Cells Behave Like a Neuronal

733 Network? Calcified Tissue International 70:435-442. DOI: 10.1007/s00223-001-1024-z.

734 Verbruggen SW, Vaughan TJ, McNamara LM. 2012. Strain amplification in bone

735 mechanobiology: a computational investigation of the in vivo mechanics of osteocytes.

736 Journal of The Royal Society Interface 9:2735-2744. DOI: 10.1098/rsif.2012.0286.

737 Wade MH, Trosko JE, Schindler M. 1986. A fluorescence photobleaching assay of gap junction-

738 mediated communication between human cells. Science (New York, N.Y.) 232:525-8. DOI:

$739 \quad 10.1126 /$ science.3961495.

740 Wang L, Dong J, Xian CJ. 2018. Computational Investigation on the Biomechanical Responses of

741 the Osteocytes to the Compressive Stimulus: A Poroelastic Model. BioMed Research

$742 \quad$ International 2018. DOI: 10.1155/2018/4071356.

743 Wang Z, Ishihara Y, Ishikawa T, Hoshijima M, Odagaki N, Ei Hsu Hlaing E, Kamioka H. 2019.

744 Screening of key candidate genes and pathways for osteocytes involved in the differential

745 response to different types of mechanical stimulation using a bioinformatics analysis. Journal of Bone and Mineral Metabolism 37:614-626. DOI: 10.1007/s00774-018-0963-7.

747 Wang Z, Odagaki N, Tanaka T, Hashimoto M, Nakamura M, Hayano S, Ishihara Y, Kawanabe N,

748 Kamioka H. 2016. Alternation in the gap-junctional intercellular communication capacity

749 during the maturation of osteocytes in the embryonic chick calvaria. Bone 91:20-29. DOI: 

10.1016/j.bone.2016.06.016.

751 Willebrords J, Maes M, Crespo Yanguas S, Vinken M. 2017. Inhibitors of connexin and pannexin 752 channels as potential therapeutics. Pharmacology \& Therapeutics 180:144-160. DOI: 753 10.1016/j.pharmthera.2017.07.001.

754 Wu X-T, Sun L-W, Yang X, Ding D, Han D, Fan Y-B. 2017. The potential role of spectrin network 755 in the mechanotransduction of MLO-Y4 osteocytes. Scientific Reports 7:40940. DOI: 756 $10.1038 /$ srep40940.

757 758

Xiong J, Onal M, Jilka RL, Weinstein RS, Manolagas SC, O’Brien CA. 2011. Matrix-embedded cells control osteoclast formation. Nature Medicine 17:1235-1241. DOI: 10.1038/nm.2448.

Xiong J, Piemontese M, Onal M, Campbell J, Goellner JJ, Dusevich V, Bonewald L, Manolagas SC, O’Brien CA. 2015. Osteocytes, not osteoblasts or lining cells, are the main source of the RANKL required for osteoclast formation in remodeling bone. PLoS ONE 10:1-19. DOI: 10.1371/journal.pone.0138189.

Xu H, Duan J, Ren L, Yang P, Yang R, Li W, Zhao D, Shang P, Jiang JX. 2018. Impact of flow shear stress on morphology of osteoblast-like IDG-SW3 cells. Journal of Bone and Mineral Metabolism 36:529-536. DOI: 10.1007/s00774-017-0870-3.

Xu X, Francis R, Wei CJ, Linask KL, Lo CW. 2006. Connexin 43-mediated modulation of polarized cell movement and the directional migration of cardiac neural crest cells. Development (Cambridge, England) 133:3629-3639. DOI: 10.1242/dev.02543.

Xu H, Wu J, Weng Y, Zhang J, Shang P. 2012. Two-dimensional clinorotation influences cellular morphology, cytoskeleton and secretion of MLO-Y4 osteocyte-like cells. Biologia 67:255262. DOI: $10.2478 / \mathrm{s} 11756-011-0161-8$.

Yang W, Harris MA, Heinrich JG, Guo D, Bonewald LF, Harris SE. 2009. Gene expression 
773 signatures of a fibroblastoid preosteoblast and cuboidal osteoblast cell model compared to the

$774 \quad$ MLO-Y4 osteocyte cell model. Bone 44:32-45. DOI: 10.1016/j.bone.2008.08.133.

775 Zhang Y, Paul EM, Sathyendra V, Davison A, Sharkey N, Bronson S, Srinivasan S, Gross TS,

776 Donahue HJ. 2011. Enhanced osteoclastic resorption and responsiveness to mechanical load

777 in gap junction deficient bone. PLoS ONE 6:e23516. DOI: 10.1371/journal.pone.0023516. 


\section{Figure 1}

Overview of the methods in this study.

(A) The continuous compressive system and (B) The overview of the experiment design. In the present study, MLO-Y4 osteocyte-like cells were proliferated under a continuous compressive force $\left(\approx 3.1 \mathrm{~Pa}\right.$ or $\left.31 \mathrm{dynes} / \mathrm{cm}^{2}\right)$ throughout the entire experiment after seeding the cells $3 \mathrm{~h}$. A long-duration ( $36 \mathrm{~h}$ ) additional continuous compressive force (CCF) at 3.2 Pa was applied as the treatment of loading history, followed by re-applying the same additional CCF $\left(\approx 3.2\right.$ Pa or 32 dynes $\left./ \mathrm{cm}^{2}\right)$ for a short duration $(1 \mathrm{~h})$, to assess the effect of loading history on the expression of interesting genes as well as the cell number, viability, morphology, and cell-to-cell communications of MLO-Y4 osteocyte-like cells. 

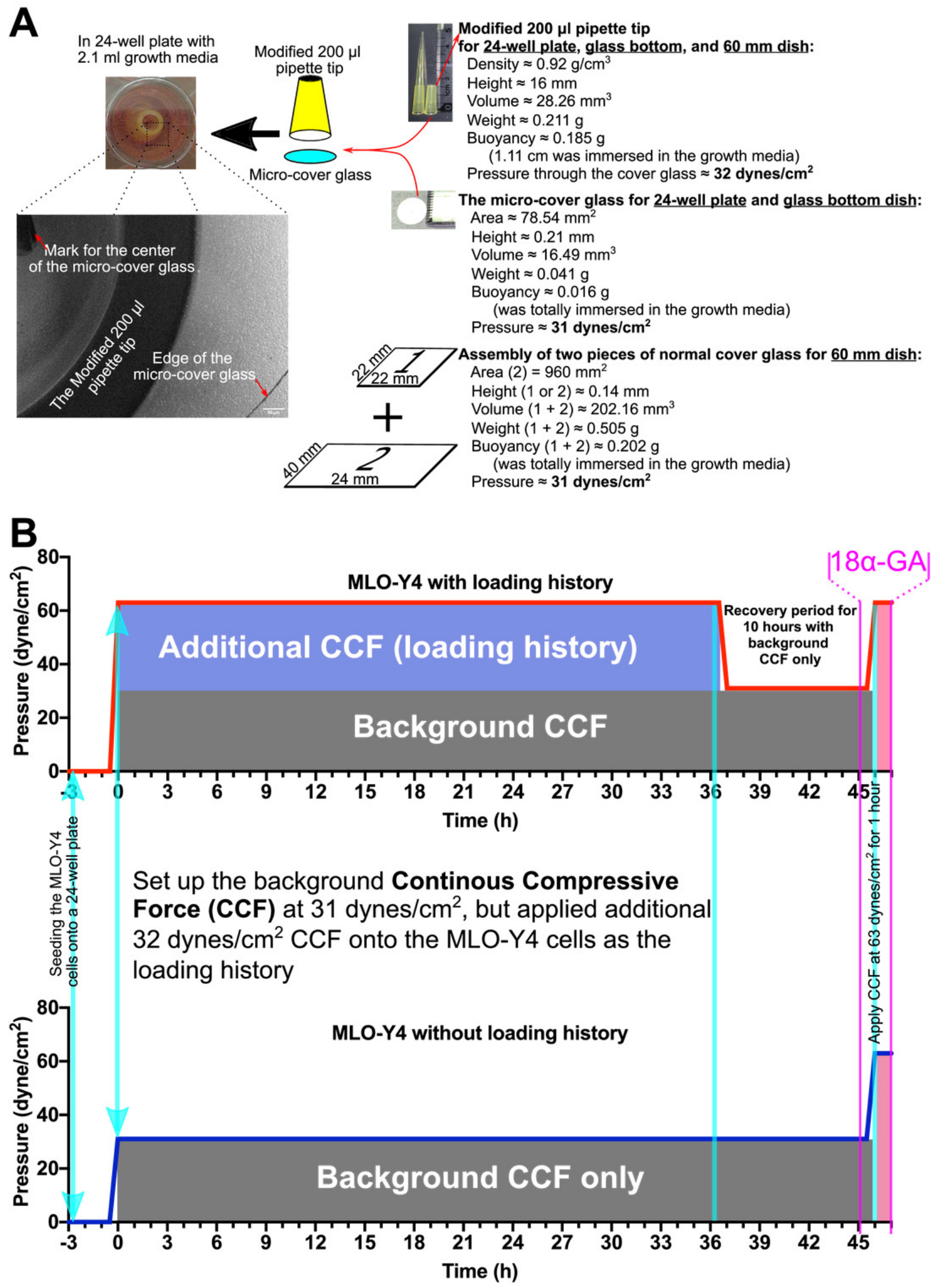


\section{Figure 2}

Calcein-AM and Hoechst 33342 staining and morphological measurements.

(A) An example of the phase-contrast image with Calcein-AM and Hoechst 33342 staining.

(B) An illustration of the morphological measurements. The right samples experienced an

additional 32 dynes $/ \mathrm{cm}^{2}$ loading before this $10 \mathrm{~h}$ recover-period as the treatment of loading history comparing to the left samples. All original images used for the morphological measurements can be downloaded from Mendeley Data (http://dx.doi.org/10.17632/2yfd2w8jfp.1\#folder-36eef903-4de0-4fcc-916a-b27262fb31a1) 

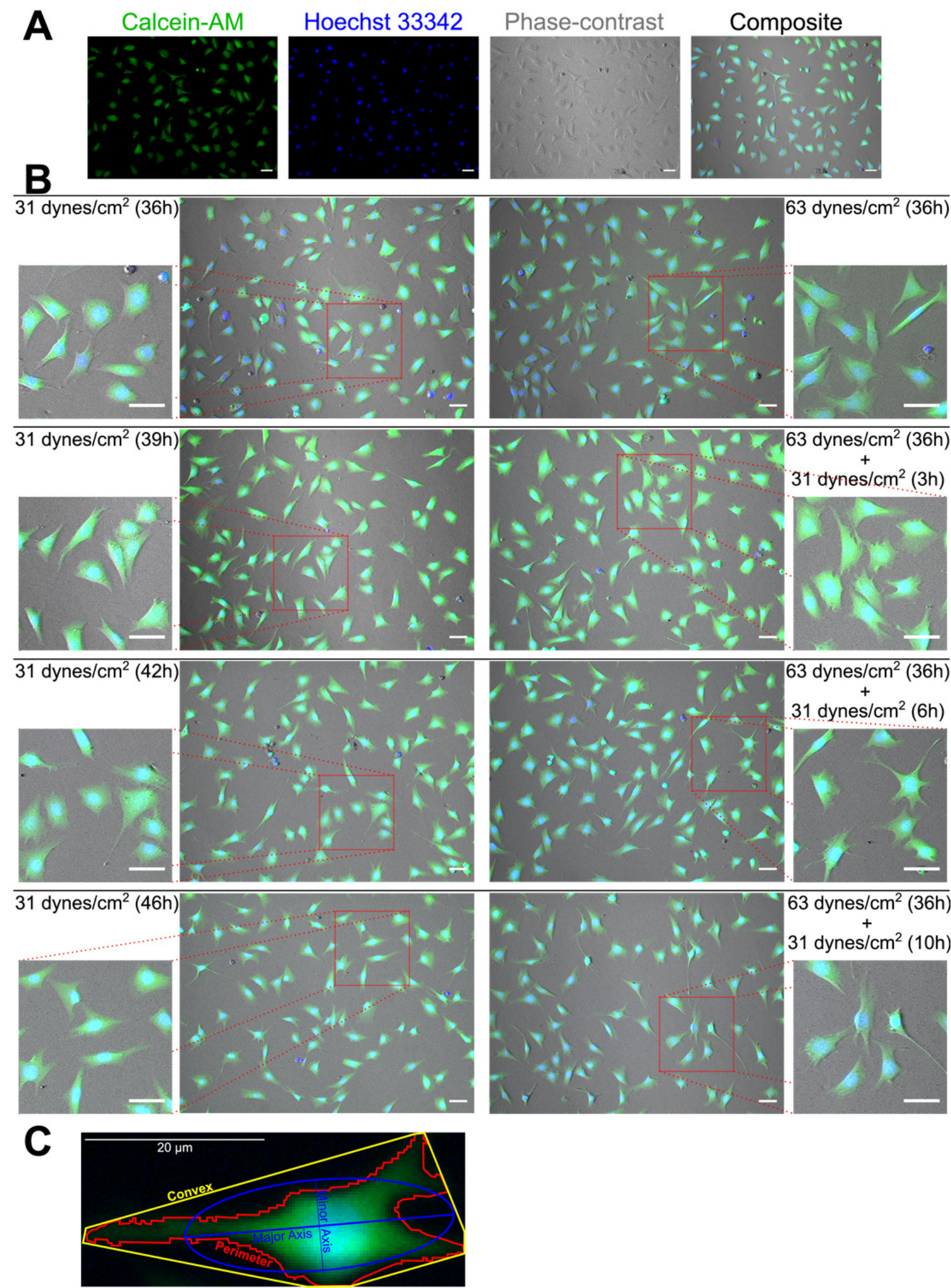

Circularity: $0.185(4 \pi \times$ Cell Area / perimeter2) Aspect Ratio: 2.973 (Major Axis / Minor Axis) Solidity: 0.570 (Cell Area / Convex Area) 
Figure 3

Proliferation of MLO-Y4 osteocytes during recovery period.

The results of cell number counting (A) and cell viability (B). ANOVA, analysis of variance; the $P$-values for each ANOVA test were listed before the legends; the $P$-values for each time point were from the independent Student's $t$-test. The values of each replicate are presented as the dots. The results are shown as mean \pm standard deviation. 
A

ANOVA:

$P>0.206 \square 31$ dynes $/ \mathrm{cm}^{2}(36 \mathrm{~h})+31$ dynes $/ \mathrm{cm}^{2}(10 \mathrm{~h})$

$P>0.099 \square 63$ dynes $/ \mathrm{cm}^{2}(36 \mathrm{~h})+31$ dynes $/ \mathrm{cm}^{2}(10 \mathrm{~h})$

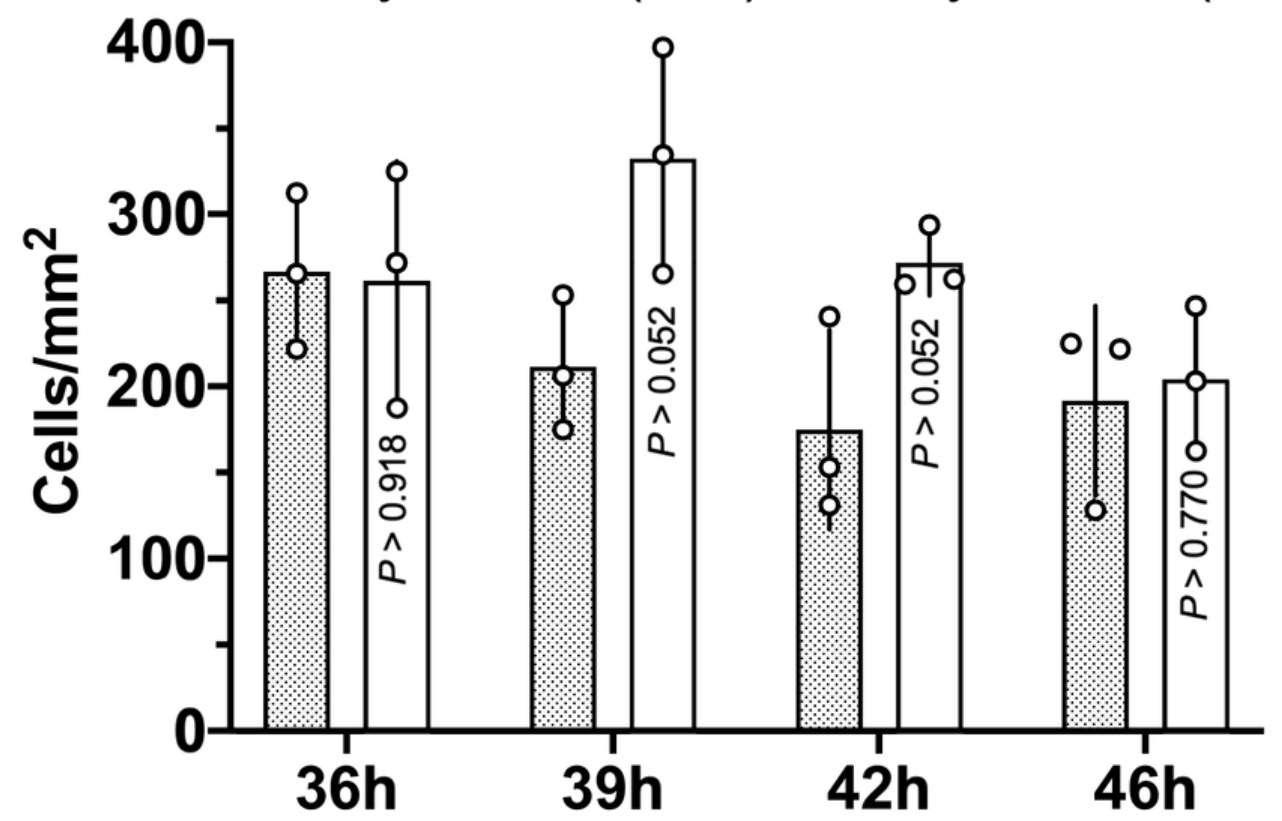

B

ANOVA:

$P>0.673 \square 31$ dynes $/ \mathrm{cm}^{2}(36 \mathrm{~h})+31$ dynes $/ \mathrm{cm}^{2}(10 \mathrm{~h})$

$P>0.150 \square 63$ dynes $/ \mathrm{cm}^{2}(36 \mathrm{~h})+31$ dynes $/ \mathrm{cm}^{2}(10 \mathrm{~h})$

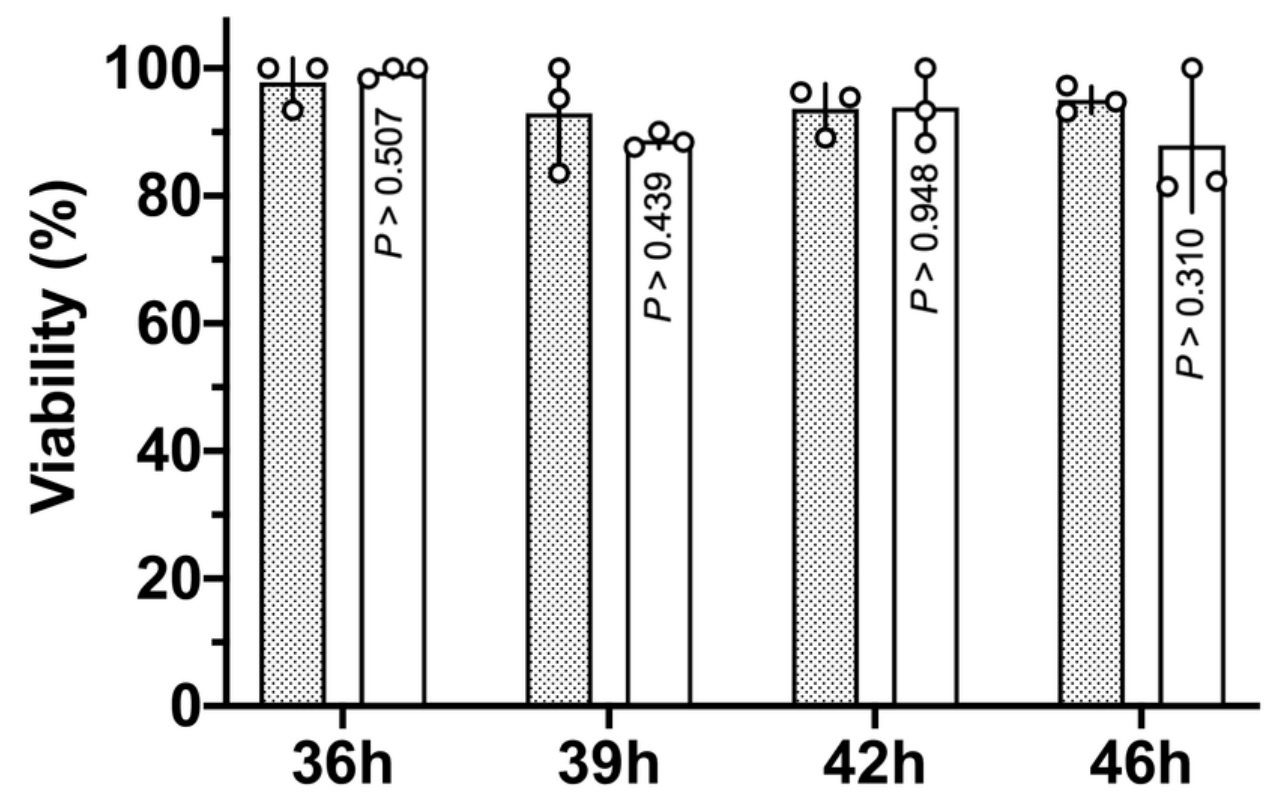




\section{Figure 4}

The results of morphological measurements during the recovery period.

The aspect ratio (A), circularity (B), and solidity (C) are shown in a violin plot. The results of each cell are represented as the dots inside the violin shape; the kernel density is represented as the outline of the violin shape; the red solid line indicates the median value; the red dashed line indicates the quartiles. FDR, false discovery rate; the FDR test was performed following the Kruskal-Wallis test. All original images used for cell morphological measurements can be downloaded from Mendeley Data (http://dx.doi.org/10.17632/2yfd2w8jfp.1\#folder-36eef903-4de0-4fcc-916a-b27262fb31a1)
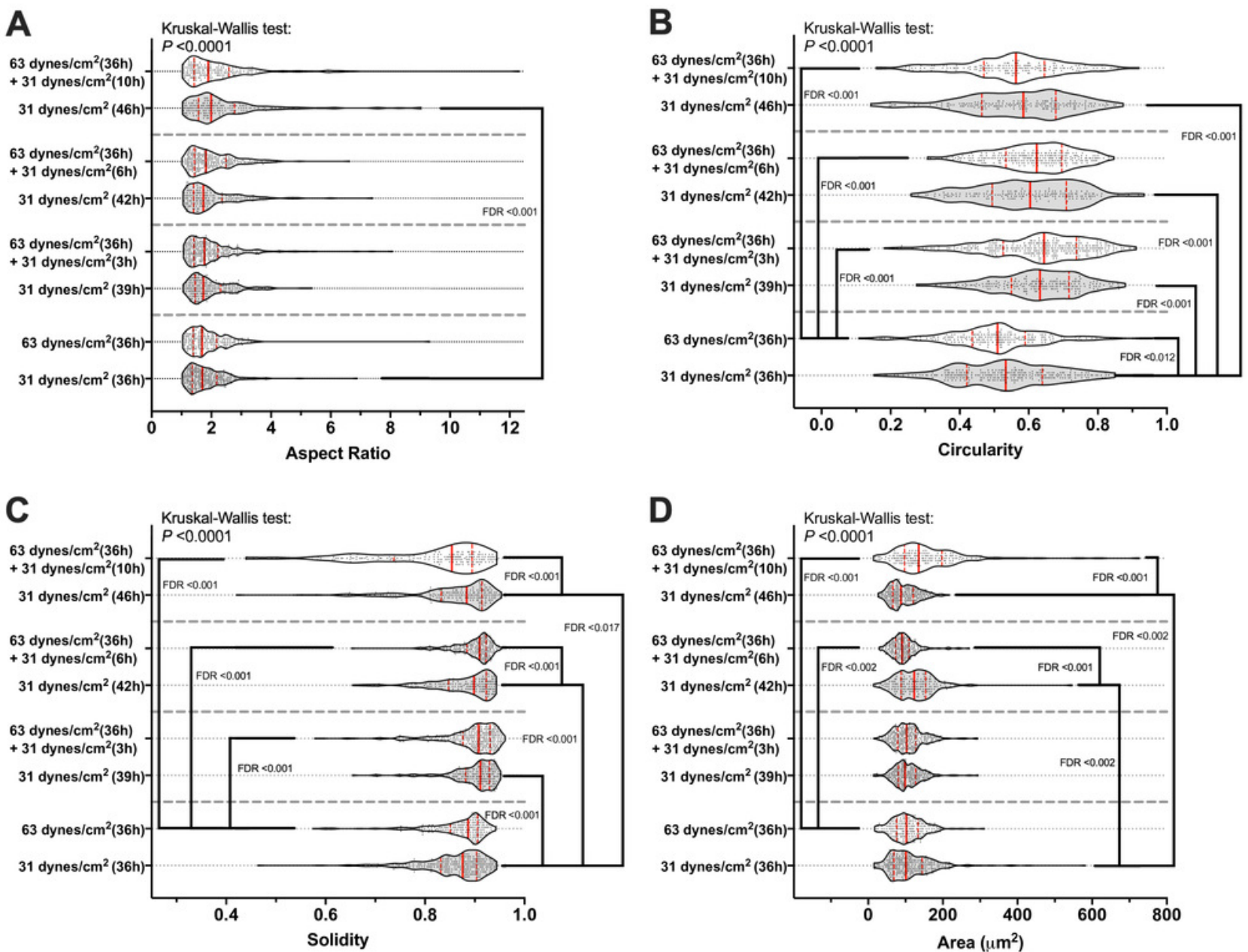


\section{Figure 5}

Expression profile of interesting genes and correlated morphological changes during the recovery period.

The mRNA expression of (A) RANKL, (B) OPG, (C) RANKL/OPG, (D) Gja1, (E) Sost, (F) Dmp1, (G) PDNP, and (H) OCN during the recovery period and the correlations of (I) OPG with Circularity, (J) of Gja1 with Circularity, and (L) of Dmp1 and Area. Independent Student's ttest was performed to test the differences of the mRNA expression of interesting genes between MLO-Y4 with and without loading history at each time point during the recovery period. *, P <0.05; **, $\mathrm{P}<0.01 ; * * *, \mathrm{P}<0.001$. The dash lines in (I), (J), (K), and (L) indicate the $95 \%$ confidence interval. The values of each replicate are presented as the dots. The results are shown as mean \pm standard deviation. 

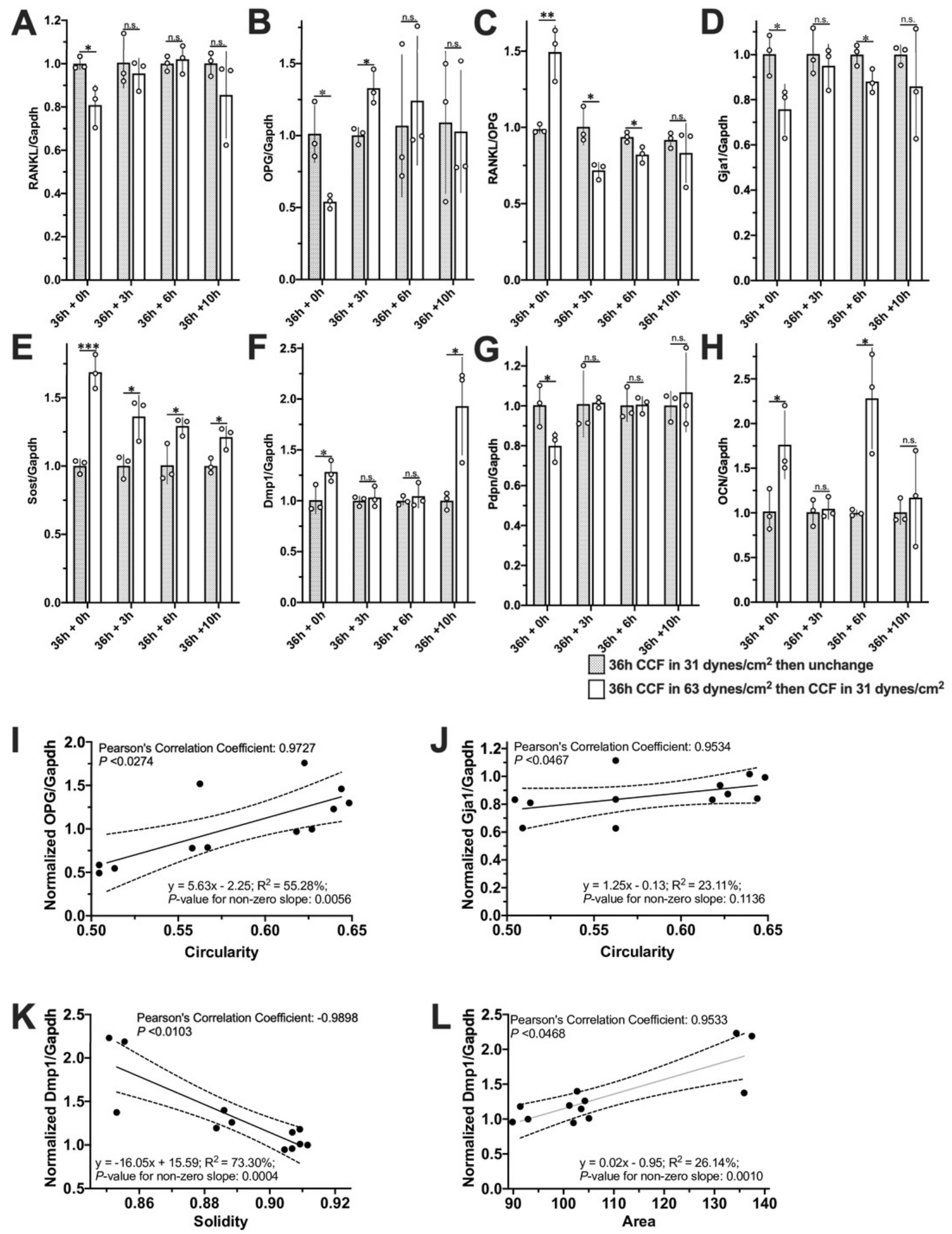
Figure 6

The influences of loading history and 18 $\alpha-G A$ on compressive force-induced changes of mRNA expression of interesting genes in MLO-Y4 osteocyte-like cells.

The mRNA expression of (A) RANKL, (B) OPG, (C) RANKL/OPG, (D) Gja1, (E) Sost, (F) Dmp1, (G) PDNP, and (H) OCN of MLO-Y4 osteocyte-like cells in response to the new applied or reapplied continuous compressive force (1h) with or without 18 $\alpha$-GA treatment (2h). The values of each replicate are presented as the dots. The results are shown as mean \pm standard deviation. ANOVA, analysis of variance; Fisher's least Significant Difference (LSD) test was performed following the ANOVA test. *, $\mathrm{P}<0.05 ; * *, \mathrm{P}<0.01 ; * * *, \mathrm{P}<0.001 ; * * * *, \mathrm{P}<0.0001$.

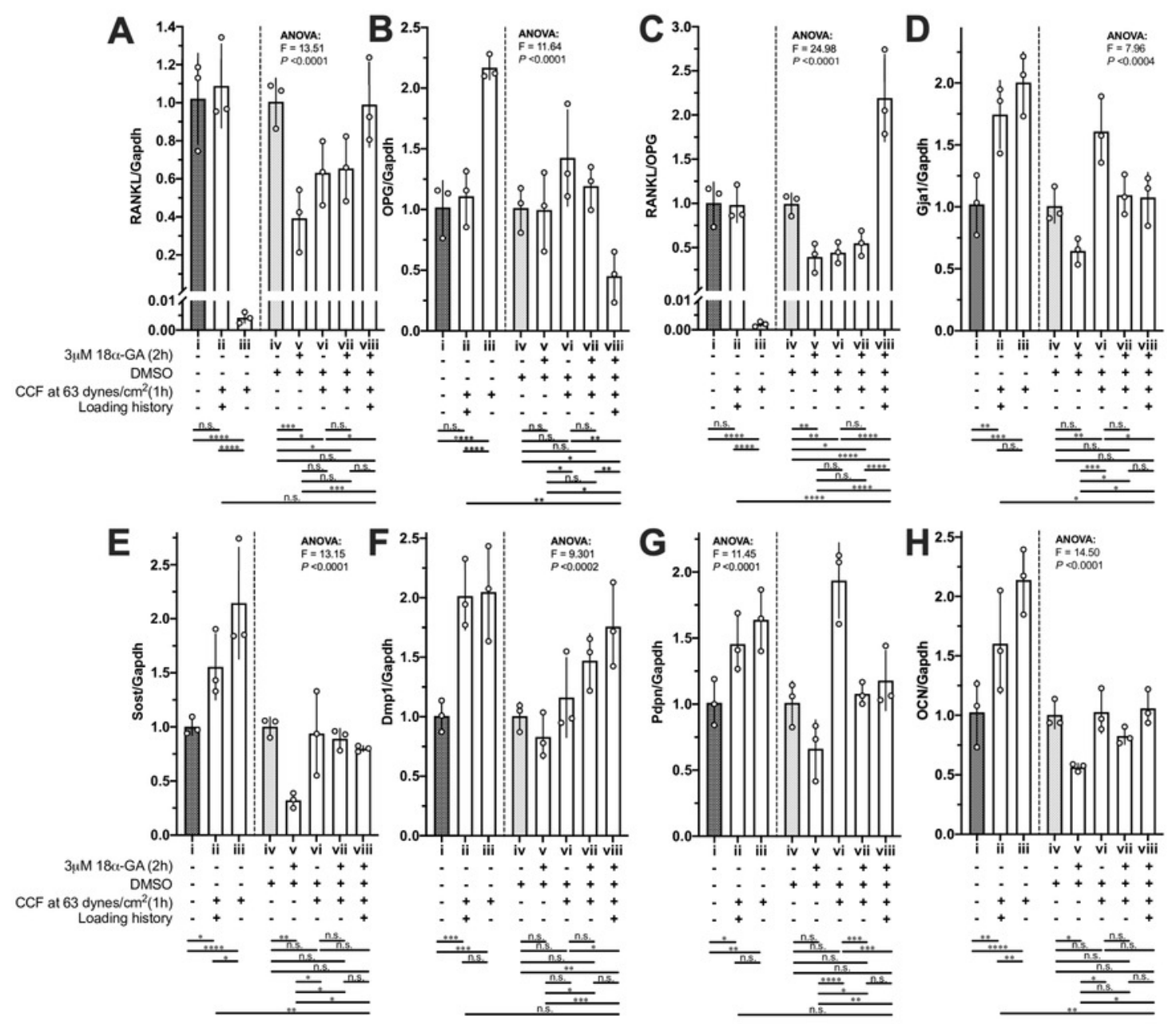




\section{Figure 7}

Cx43 expression and fluorescence recovery after photobleaching (FRAP) assay in MLOY4 osteocyte-like cells

(A) The expression of $\mathrm{Cx} 43$ in normal cultured (without cover glass) MLO-Y4 osteocyte-like cells. (B) The instrument scheme and examples for FRAP assay in MLO-Y4 osteocyte-like cells. (C) FRAP recover curve and fitted model of calcein fluorescence transportation in MLOY4 osteocyte-like cells with or without loading history. After a two-way ANOVA test, multiple testing with false discovery rate (FDR) control for the recover curves was performed and transformed FDR values are plotted (higher plot bar with lower original FDR value). The (D) predicted mobile fraction, (E) coefficient $k$, and $(\mathbf{F})$ goodness of fit from the fitted model were investigated. (G) The number of neighbouring cells surrounding the photobleached target cell were counted. All original time-lapse images used for FRAP analysis can be downloaded from Mendeley Data (DOI:

http://dx.doi.org/10.17632/2yfd2w8jfp.1\#folder-177a56ec-cb22-492b-8f72-21438f23394c). In (D), (E), (F), and (G), Fisher's least Significant Difference (LSD) test was performed following the ANOVA test, the values of each replicate are presented as the dots, and the results are shown as mean \pm standard deviation. The results in (C) are shown as the mean \pm standard error of the mean. Scale bar $=20 \mu \mathrm{m}$. ANOVA, analysis of variance; $*, P<0.05 ; * *, P<0.01$. 

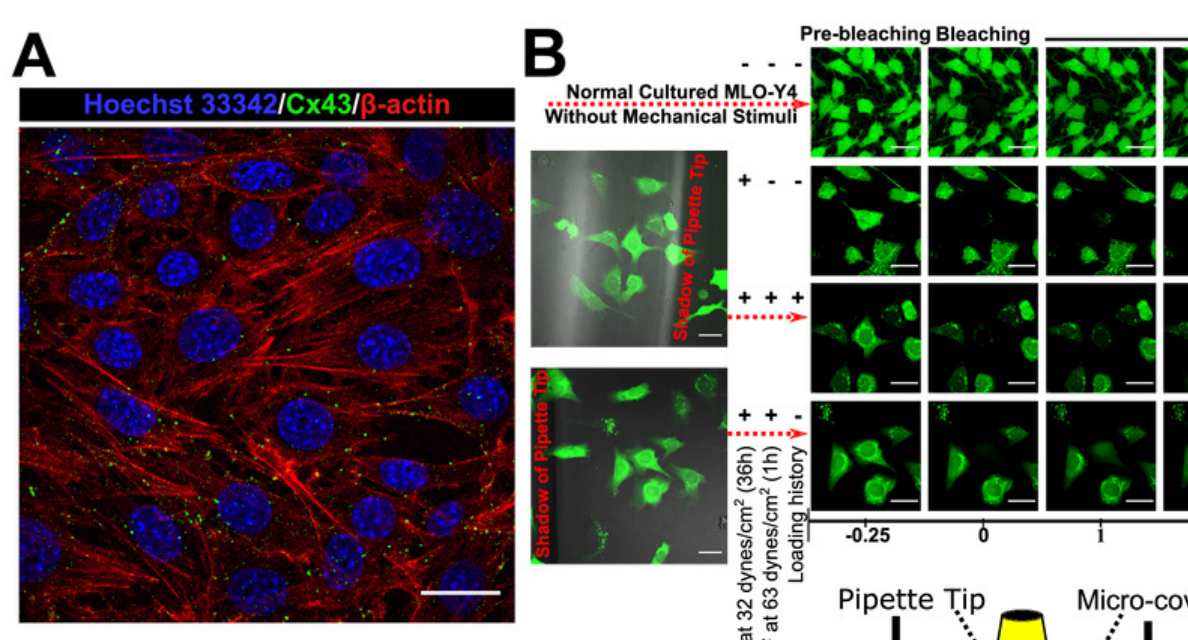

Post-bleaching
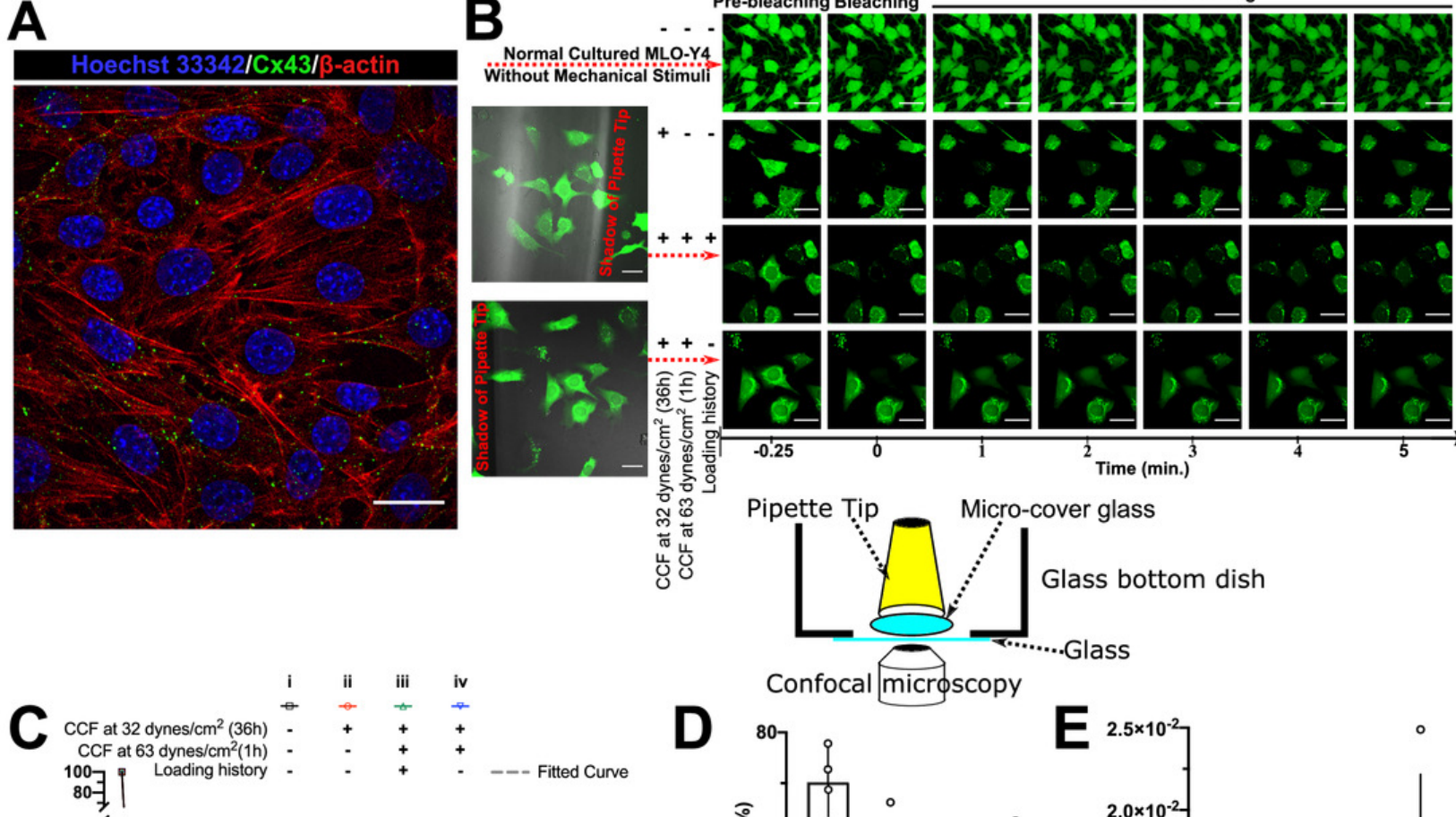

Confocal microscopy
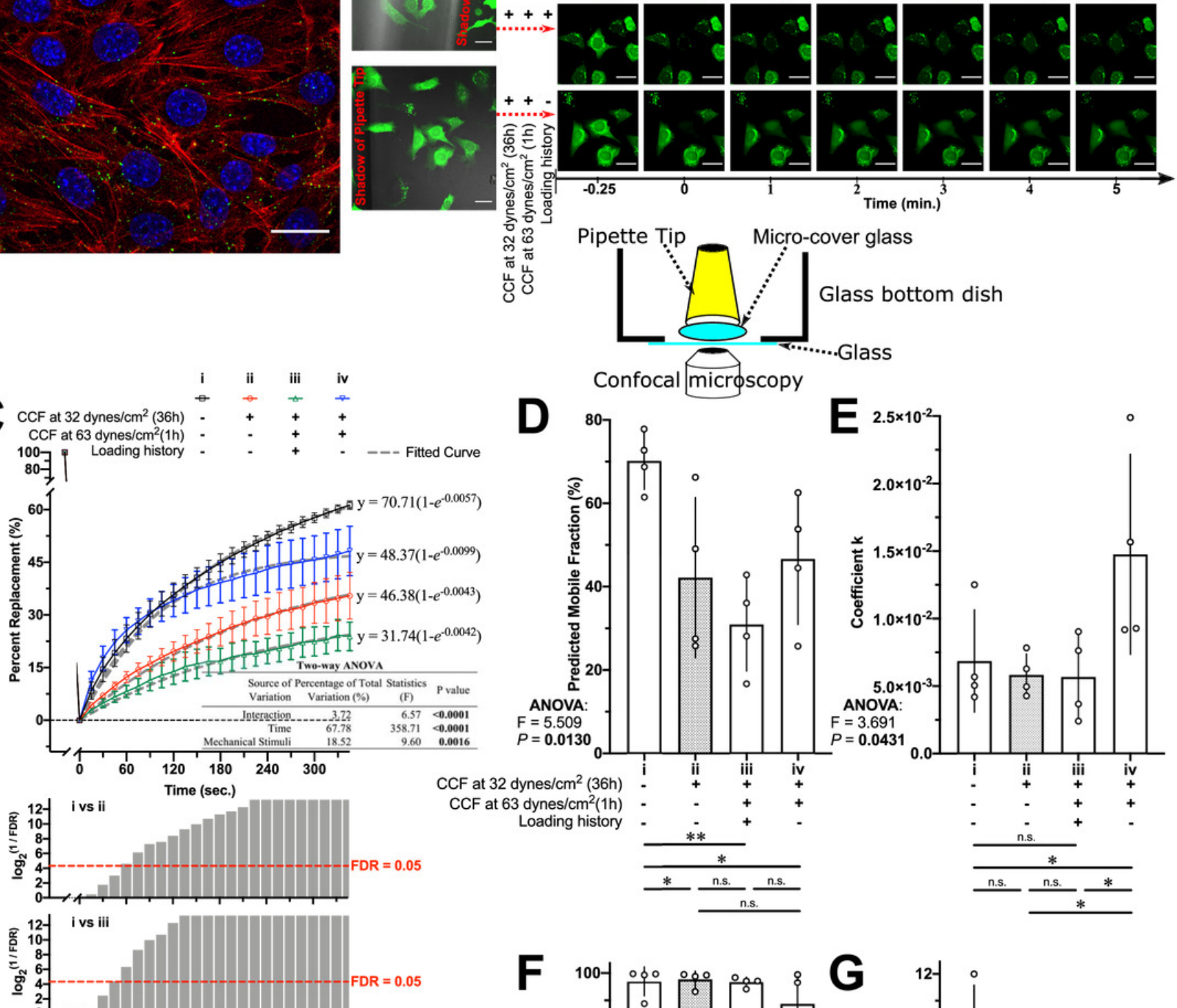

Pipette Tip Mipro-cover glass
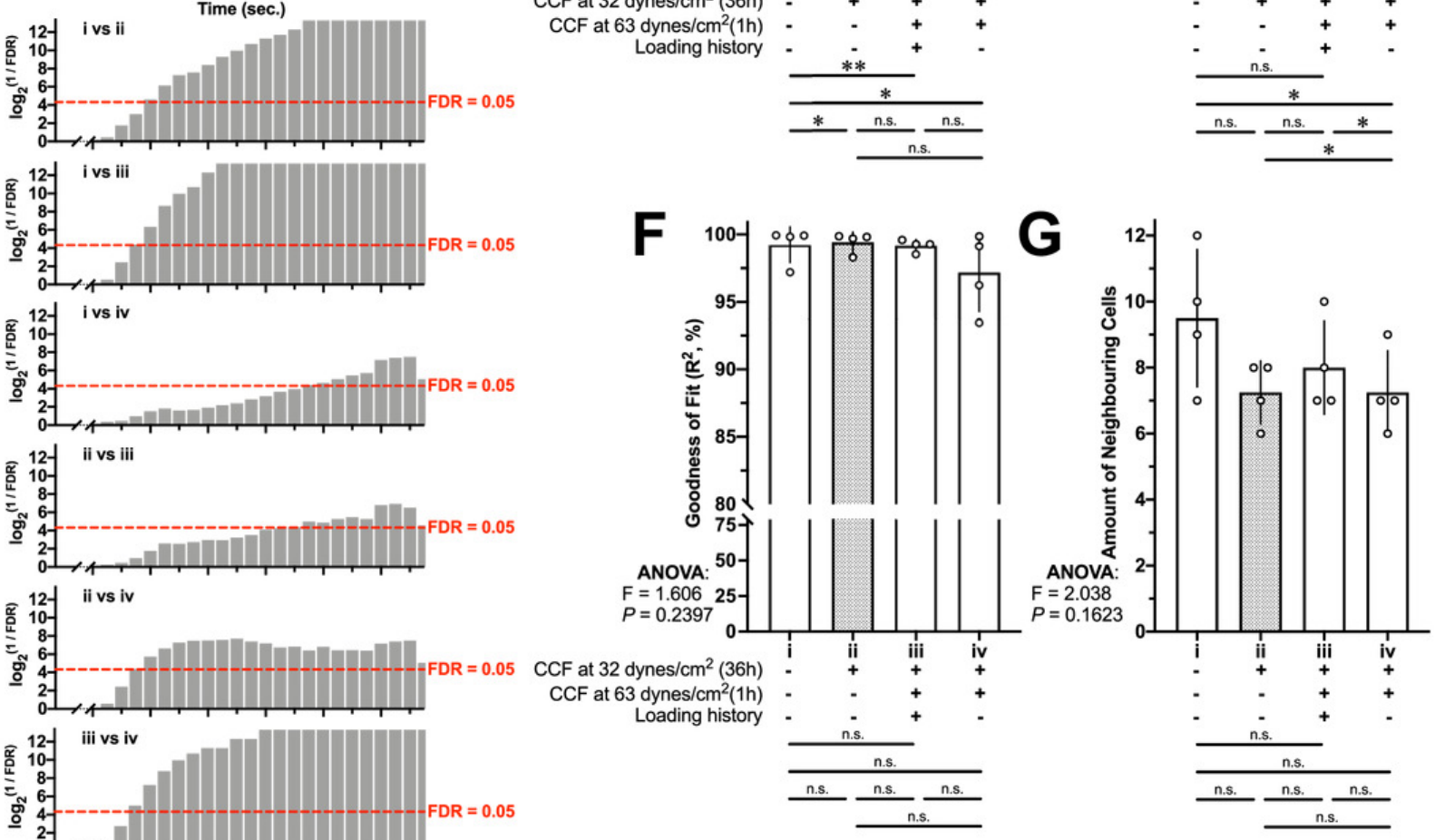

12- ivs iv

政

$\begin{array}{cc} & 8 \\ \text { : } & 6-1 \\ \text { 은 } & 4\end{array}$

12- iivs iii

$\bar{x}$
$12-10$
$8-1$

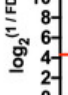

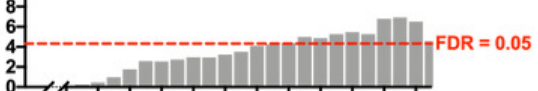

( 12 iivs iv

$12-1$
8
8

을 $6-1$

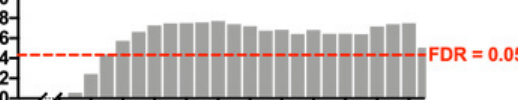

12- iiivs iv

商 $12-10-$

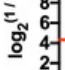

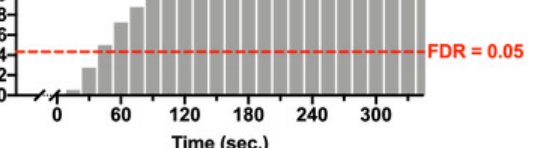




\section{Figure 8}

Protein expression changes of $\mathrm{Cx} 43, \mathrm{pC} \times 43$, and Sost of MLO-Y4 osteocyte-like cells in response to re-applied compressive force with or without loading history.

(A) Loading history suppressed the mechanosensitivity of $\mathrm{Cx} 43$ and $p \mathrm{C} \times 43$ but not Sost in response to the compressive force applied again. The quantitative results of (A) for protein expression of (B) Cx43, (C) pCx43, (D) pCx43/Cx43, and (E) Sost. Multiple testing with false discovery rate (FDR) control followed a one-way ANOVA test was performed by using the results from two independent replications. The values of each replicate are presented as the dots. The results are shown as mean \pm standard deviation. ANOVA, analysis of variance. *, FDR $<0.05 ; * *, F D R<0.01 ; * * *, F D R<0.001$. The molecular weight (MW) was estimated by a standard curve of the logarithm of the MW versus relative migration distance that was generated using the Precision ${ }^{\mathrm{TM}}$ Plus Protein Dual Xtra Standards. 
A
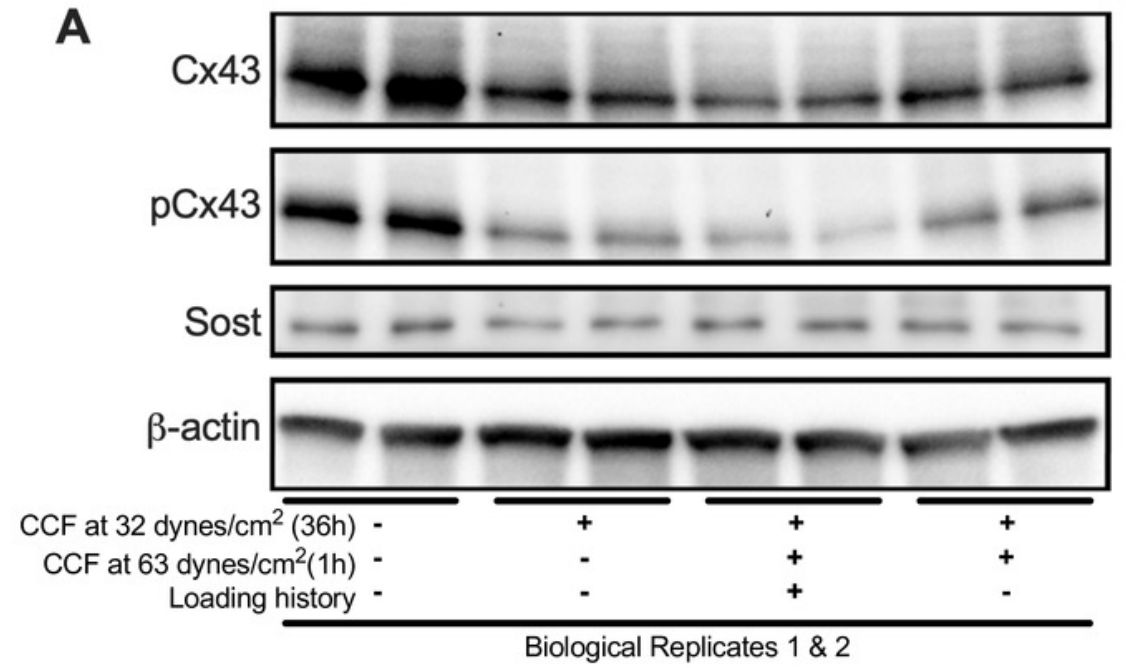

Biological Replicates 1 \& 2
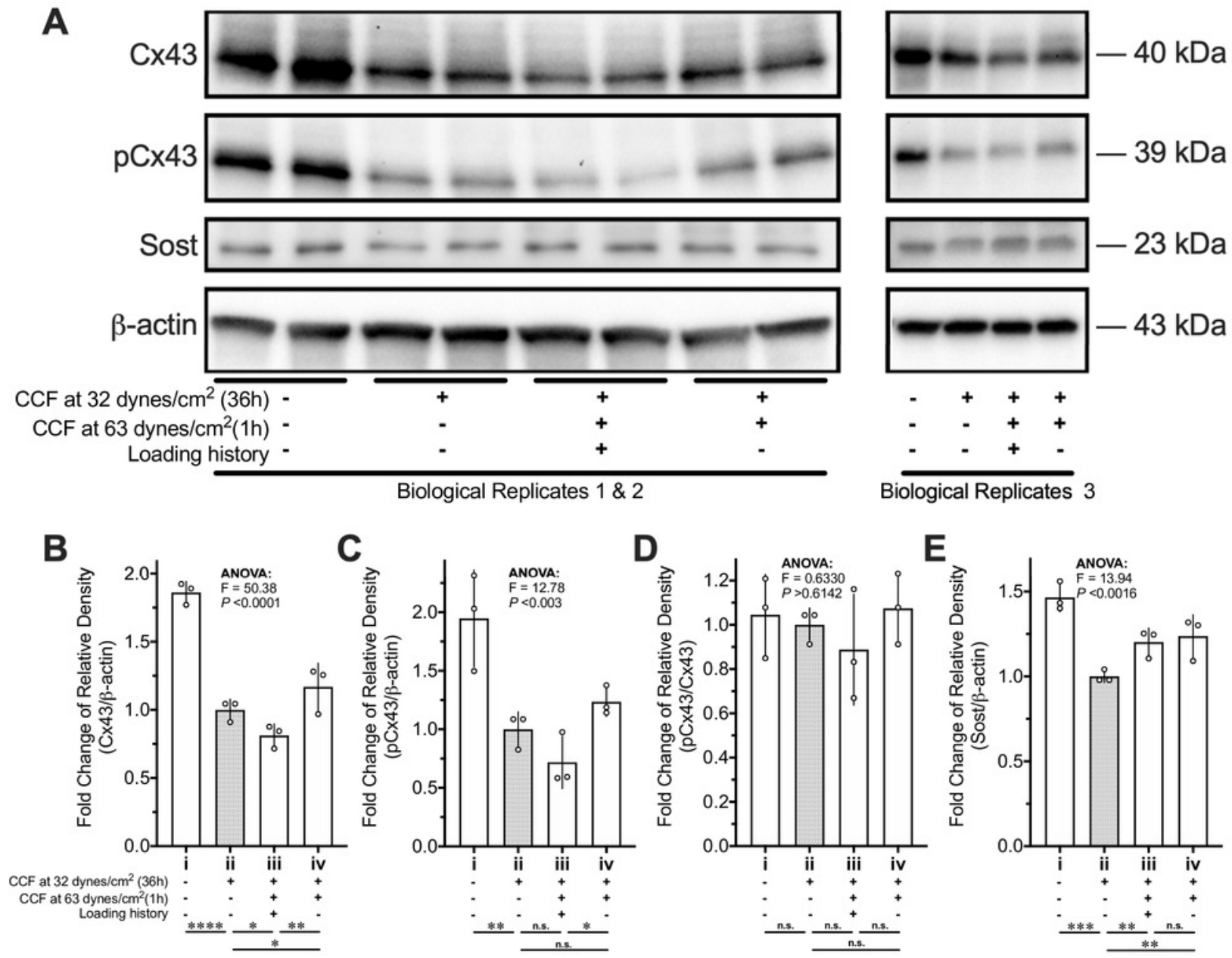